\title{
Toggles, Dollar Signs, and Triangles: How to (In)Effectively Convey Privacy Choices with Icons and Link Texts
}

\author{
Hana Habib* \\ htq@andrew.cmu.edu \\ Carnegie Mellon University \\ Pittsburgh, PA, USA \\ Alessandro Acquisti \\ acquisti@andrew.cmu.edu \\ Carnegie Mellon University \\ Pittsburgh, PA, USA
}

\author{
Yixin Zou ${ }^{*}$ \\ yixinz@umich.edu \\ University of Michigan \\ Ann Arbor, MI, USA \\ Lorrie Faith Cranor \\ lorrie@cmu.edu \\ Carnegie Mellon University \\ Pittsburgh, PA, USA
}

\author{
Yaxing Yao \\ yaxingy@andrew.cmu.edu \\ Carnegie Mellon University \\ Pittsburgh, PA, USA \\ Joel R. Reidenberg \\ Fordham University \\ New York, NY, USA
}

\author{
Norman Sadeh \\ ns1i@andrew.cmu.edu \\ Carnegie Mellon University \\ Pittsburgh, PA, USA
}

\author{
Florian Schaub \\ fschaub@umich.edu \\ University of Michigan \\ Ann Arbor, MI, USA
}

\begin{abstract}
Increasingly, icons are being proposed to concisely convey privacyrelated information and choices to users. However, complex privacy concepts can be difficult to communicate. We investigate which icons effectively signal the presence of privacy choices. In a series of user studies, we designed and evaluated icons and accompanying textual descriptions (link texts) conveying choice, opting-out, and sale of personal information - the latter an opt-out mandated by the California Consumer Privacy Act (CCPA). We identified icon-link text pairings that conveyed the presence of privacy choices without creating misconceptions, with a blue stylized toggle icon paired with "Privacy Options" performing best. The two CCPA-mandated link texts ("Do Not Sell My Personal Information" and "Do Not Sell My Info") accurately communicated the presence of do-notsell opt-outs with most icons. Our results provide insights for the design of privacy choice indicators and highlight the necessity of incorporating user testing into policy making.
\end{abstract}

\section{CCS CONCEPTS}

- Security and privacy $\rightarrow$ Usability in security and privacy; • Human-centered computing $\rightarrow$ Usability testing; • Social and professional topics $\rightarrow$ Governmental regulations.

\section{KEYWORDS}

Privacy, choice, icon design, CCPA.

${ }^{*}$ Hana Habib and Yixin Zou contributed equally to this research.

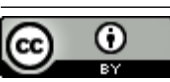

This work is licensed under a Creative Commons Attribution International 4.0 License.

CHI '21, May 8-13, 2021, Yokohama, Japan

(c) 2021 Copyright held by the owner/author(s).

ACM ISBN 978-1-4503-8096-6/21/05.

https://doi.org/10.1145/3411764.3445387

\section{ACM Reference Format:}

Hana Habib, Yixin Zou, Yaxing Yao, Alessandro Acquisti, Lorrie Faith Cranor, Joel R. Reidenberg, Norman Sadeh, and Florian Schaub. 2021. Toggles, Dollar Signs, and Triangles: How to (In)Effectively Convey Privacy Choices with Icons and Link Texts. In CHI Conference on Human Factors in Computing Systems (CHI '21), May 8-13, 2021, Yokohama, Japan. ACM, New York, NY, USA, 25 pages. https://doi.org/10.1145/3411764.3445387

\section{INTRODUCTION}

Notice and choice are common components of privacy regulation and consumer protection guidelines. They are intended to communicate how companies handle consumer data and to give consumers control over the collection and use of their personal information. Unfortunately, the mechanisms that websites commonly use to provide privacy notices and choices are fraught with issues. Privacy policies are lengthy [22,77] and full of jargon [34]. Privacy choices are difficult to find, as their location varies across websites [47, 48]. Privacy advocates, legal experts, and academic researchers have argued for standardized mechanisms to provide privacy notices and choices $[2,18,101]$. Requirements that privacy notices and choices be clear and accessible have also emerged in recent regulation, such as the California Consumer Privacy Act (CCPA) [88] and Europe's General Data Protection Regulation (GDPR) [33]. Researchers have explored ways to help consumers find and understand privacyrelated information and choices. Examples include privacy dashboards [44], certifications [9], scores [45, 98], labels [30, 63, 64], pop-ups [84, 119], as well as icons [6, 27, 28, 50, 55, 81, 102].

In principle, icons can communicate concepts quickly and concisely across linguistic and cultural differences [58]. Icons can be recognized and memorized more easily than other UI elements with richer information [102]. However, privacy concepts can be difficult to convey through icons $[6,28,101,105]$. Prior attempts at developing icons have primarily focused on conveying information about data flows or specific data practices (e.g., [6, 27, 81, 102]). The concept of choice has been less explored in previous privacy iconography research - even though privacy choices are a key component of consumer privacy regulation $[18,33,88]$. 
Our study investigates how to effectively convey to consumers the presence of privacy choices on websites through icons and accompanying descriptions (which we refer to as link texts). In particular, we consider the presence of generic privacy choices and an opt-out for the sale of personal information, as mandated by the CCPA. We first developed 11 icons that center on three choicerelated concepts: the broad idea of choice, the action of opting-out, and choices regarding the sale of personal information, before selecting five icons for further refinement and evaluation. Because icons - especially new ones - are rarely fully self-explanatory [50], we further evaluated 16 link texts to accompany the icon, including two link texts mandated by the CCPA. We then conducted a nearly full-factorial online experiment $(n=1,468)$ to assess how well different combinations of the most promising icons and link texts from the pre-studies communicated the presence of privacy or do-notsell choices. Finally, we conducted an experiment to test an icon that the California Attorney General's Office (OAG) proposed for the CCPA opt-out [91] after we shared our initial results with them.

Our research provides valuable insights into the design of privacy choice indicators. Through an iterative process, we identified promising icon and link text pairings that effectively indicate privacy choices to consumers. A blue stylized toggle icon best conveyed the idea of choices, whereas icons focused on the sale of personal information created misconceptions about what would happen after clicking the icon. The Digital Advertising Alliance's Privacy Rights icon [112] and the older AdChoices icon [113], as comparison points for our newly designed icons, suggested "more information" but not "choice." For icon-text combinations, "Privacy Options" paired with the blue stylized toggle icon best conveyed the presence of privacy choices. The link texts mandated by the CCPA ("Do Not Sell My Personal Information" and "Do Not Sell My Info") effectively conveyed the expectation of choices related to the sale of personal information in combination with most icons. Our follow-up study of the OAG's icon revealed that even minor design changes could severely reduce an icon's comprehension and increase misconceptions.

\section{BACKGROUND \& RELATED WORK}

We first summarize legal and self-regulatory requirements regarding privacy choices. We then discuss usability issues with mechanisms for conveying privacy choices.

\subsection{Requirements for Privacy Choices}

The GDPR requires businesses to provide privacy choices to European consumers, including an option to request the erasure of personal data about them (Art. 17) and opt-outs for data processing for direct marketing purposes (Art. 21) [33]. The GDPR also emphasizes the usability of privacy notices and choices, requiring that notices be provided in "a concise, transparent, intelligible and easily accessible form, using clear and plain language” (Art. 12). In the United States, privacy choices are regulated by sector-specific federal laws and state privacy laws, such as the CAN-SPAM Act's requirement of marketing email opt-outs [35], and the Children's Online Privacy Protection Act (COPPA)'s requirement of honoring parental requests about data collection and deletion for children under 13 [36]. Since January 2020, the CCPA provides California residents the right to opt out of the sale of their personal information by companies [88]. The first draft of the proposed CCPA regulations specified that this opt-out should be provided, at a minimum, through "an interactive form accessible via a clear and conspicuous link titled 'Do Not Sell My Personal Information,' or 'Do Not Sell My Info, ${ }^{1}$ on the business's website or mobile application" as well as an unspecified optional "opt-out button or logo" [88]. In November 2020, Californians voted to pass the California Privacy Rights Act (CPRA) [89], which amends and expands consumer privacy rights stipulated by the CCPA.

Self-regulatory requirements exist for online advertising practices [24, 53, 82]. Since 2010, the Digital Advertising Alliance (DAA) has required its member companies to provide opt-outs for trackingbased targeted advertising by placing the AdChoices icon (see Table 1) and an approved text above an ad [24]. The DAA recently introduced a Privacy Rights icon (a green variant of the AdChoices icon; see Table 1), to address the CCPA's opt-out requirements [112]. Additionally, the Interactive Advertising Bureau Europe has published the Transparency and Consent Framework for obtaining consumer consent under the GDPR [54].

Companies' compliance with legal and self-regulatory requirements varies. Opt-outs for email communications are common due to the CAM-SPAM Act, with most US companies offering links to unsubscribe within email messages and privacy policies [23, 48]. However, privacy choices related to targeted advertising or data deletion are less common $[46,48,94]$. Even when they exist, privacy choices appear at inconsistent locations, and often exhibit unhelpful information as well as broken links [48]. Furthermore, research on the CCPA's do-not-sell provision has shown that consumers struggle to locate the required links to opt out, and the opt-out processes are permeated with dark patterns [75, 87]. As such, consumers face considerable barriers in exercising privacy choices [23, 47, 48, 67, 94].

\subsection{Communicating Privacy Choices}

Privacy choices are often disclosed in privacy policies. However, research has shown that most users do not read privacy policies [80, 86] or struggle to comprehend them due to vague descriptions and jargon $[11,59,79,100]$. Given the estimated time required to peruse privacy policies on visited websites, it would be unrealistic to expect users to read them routinely [77]. These findings suggest the need for alternative privacy notices that make privacy information more accessible and understandable [106]. Examples of such alternatives include privacy dashboards [44], privacy certifications and seals [9], privacy grades and scores $[29,45,61,98]$, privacy labels [31, 63, $65,117]$, consent banners and pop-ups [74, 84, 119], and privacy icons $[50,55,81,102]$.

Privacy dashboards allow consumers to inspect the data companies have collected about them and adjust their privacy settings [101]. For example, the browser extension Ghostery provides an interface for users to learn which web trackers are present on visited websites and block or permit certain trackers [44], though users may struggle to comprehend information about trackers [107].

\footnotetext{
1 "Do Not Sell My Info" was mandated in the proposed CCPA regulations [88] but got eliminated in the final version [90] after we completed our study.
} 
Privacy seals and certifications, such as the Enterprise Privacy Certification by TrustArc (formerly TRUSTe) [9], are designed to signal that businesses comply with legal requirements or industry standards [101]. Privacy grades and scores indicate how well websites protect their users' privacy through numeric ratings, e.g., ToS;DR [61], Privacy Finder [29, 45], and PrivacyGrade.org for mobile apps [98]. Privacy labels, similar to food nutrition labels, help users quickly learn about and compare privacy-related attributes of products or services, including websites [63, 64], Internet of Things devices [30, 31], search results [14, 117], and mobile apps [3, 65]. Privacy choices, mostly related to cookie management, are also presented in consent pop-ups and banners on websites [22], but often provide users with limited choices and nudge them to accept tracking [74, 84, 119].

\subsection{Privacy Icons}

Researchers have proposed various privacy icons as succinct indicators of complex privacy concepts. Some privacy icons represent specific data practices, such as Disconnect.me's icons for different types of tracking [27] and Mozilla's icons for retention periods and third-party data sharing and use [81]. Some only serve specific application domains, such as social media [55], web links [62], or webcams [28, 97], while others can apply across contexts [50]. Icons are also commonly used as security indicators (e.g., a lock in a browser's URL bar that indicates HTTPS [37]). However, prior work has found that users tend to ignore or misunderstand these indicators [41, 72, 108]. Fewer privacy icons are designed to convey privacy choice, consent, or opt-outs. The Stanford Legal Design Lab has proposed icons [111] that could potentially indicate privacy choices, but they have not been empirically evaluated. While the Data Protection Icon Set (DaPIS) [102] has been user-tested, it is specific to GDPR consumer privacy rights.

Icons have several advantages that can address the limitations of traditional privacy notices. Icons can visually communicate information concisely while circumventing language and cultural barriers [76]. Icons can be useful information markers since they are easy to recognize $[12,51]$. When placed next to lengthy privacy statements, icons can enhance readability by helping users navigate the text [102]. In a review of iconography guidelines, Bühler et al. [12] summarized principles for effective icons - they should be based on users' knowledge and needs, utilize well-known concepts, and closely mimic real-world objects. However, designing comprehensible icons is challenging. Icons alone sometimes perform worse than text-only or icon-text interfaces in assisting learning [122]. Fischer-Hübner et al. [38] therefore argue that icons should be used alongside text to illustrate data practices in privacy policies and aid user comprehension. Beyond an icon's comprehensibility, the focus of our study, discoverability is another challenge. For instance, the size, position, state, and color all impacted how visible the AdChoices icon was to users on a mobile device [42].

Privacy icons explored in prior work have primarily focused on communicating data practices, but few proposed privacy icons have received wide adoption. Even widely adopted icons, such as DAA's AdChoices icon, are problematic [42, 78, 118]. Not much work has focused on using icons to convey privacy choices effectively to consumers. We fill this gap by iteratively designing and evaluating privacy choice icons and associated link texts. Complementing prior research on icons for GDPR-specific user rights [102], we focus on conveying the presence of general privacy choices, as well as the CCPA-mandated do-not-sell opt-out.

\section{STUDY OVERVIEW}

Between November 2019 and February 2020, we conducted a series of studies to iteratively design and evaluate two types of icons and associated link texts: one indicating the presence of generic privacy controls on websites, and the other indicating choices related to the sale of personal information, as required by the CCPA. Our research involved two pre-studies (one focusing on icons and the other on link texts), a large-scale online experiment to evaluate icon-link text combinations, and a follow-up evaluation of an icon that the Office of the California Attorney General (OAG) had proposed based on our initial findings.

Icon Pre-Study (Section 4, $n=520$ ) We developed 11 privacy icons that center on three choice-related concepts: the broad idea of choice, the action of opting out, and choices regarding the sale of personal information. We iteratively refined and tested these icons to identify which to include in our main experiment. Our icon pre-study suggests that a stylized toggle switch was promising for conveying the presence of choice; three icons that included dollar signs, slashes, stop signs, and ID cards were good candidates for conveying the CCPA do-not-sell opt-out.

Link Text Pre-Study (Section 5, $n=540$ ) We tested 16 textual descriptions, or link texts, to accompany the icons we developed. We analyzed how each link text, when displayed alone, was interpreted by participants; and identified three link texts ("Privacy Options," "Privacy Choices," and "Personal Info Choices") with mostly correct interpretations. The two CCPA link texts ("Do Not Sell My Personal Information" and "Do Not Sell My Info") effectively indicated choices related to the sale of personal information, but did not generalize to broader privacy-related choices.

Icon-Text Combinations Evaluation (Section 6, $n=1,468$ ) We conducted a large-scale, nearly full-factorial online experiment to evaluate how well 23 combinations of icons and link texts, selected from our pre-studies, communicated the presence of privacy choices and do-not-sell choices. We showed participants one icontext combination on a screenshot of a fictitious online shoe retailer webpage, mimicking how users may see such privacy choice indicators in the real world. A blue stylized toggle icon paired with the link text "Privacy Options" best conveyed the presence of privacy choices. The two CCPA link texts effectively conveyed the presence of do-not-sell opt-outs when paired with most icons.

OAG Icon Evaluation (Section 7, $n=421$ ) After we shared our results with the OAG, they proposed an icon for the CCPA's do-notsell opt-out, which was similar to our stylized toggle icon but with notable deviations. We conducted a follow-up experiment to explore the impact of the icon's toggle style and color on expectations for donot-sell choices. Compared to our stylized toggle icon, participants were much more likely to perceive the OAG's proposed icon as a toggle switch rather than a static icon. ${ }^{2}$

\footnotetext{
${ }^{2}$ In December 2020, the OAG published the fourth set of modifications to the CCPA regulations [92], recommending that businesses use our blue stylized toggle icon next to the CCPA link text when notifying consumers of their right to opt out of the sale of
} 


\section{ICON PRE-STUDY}

We developed 11 icons related to privacy choices and evaluated how users interpreted the icons with and without a text description. We found that a stylized toggle icon effectively communicated the concept of choice, but communicating the concept of "privacy choice" was difficult without text. While icons with arrows to depict removal were mostly unsuccessful, icon elements focusing on "do not" and "sell" could communicate an opt-out for the sale of personal information. However, participants often misunderstood an icon without a text description.

\subsection{Icon Development}

4.1.1 Icon ideation. To explore potential icon candidates, we leveraged existing privacy iconography to generate three key concepts in line with our objectives: the broad concept of choice, the action of opting out, and a specific opt-out related to the sale of personal information for the CCPA. We did not attempt to design an icon that visualizes privacy since privacy is a broad concept with many interpretations [85]. Additionally, we did not test existing privacy and security icons since they are already known for representing other concepts unrelated to privacy choices (e.g., lock or shield for HTTPS indicator [37]), or focus on specific data practices [102].

To capture a wide range of icon ideas embodying the three choicerelated concepts we identified, we conducted design ideation activities at our institutions with colleagues interested in privacy and security research. During the activities, participants drew ideas on sticky notes and discussed themes with the group. We then conducted affinity diagramming [70] of the sketches by grouping similar ideas and identifying themes in the visual elements participants used to represent the three concepts (see Figure 1). In selecting themes to iterate upon further, we eliminated those focusing on privacy more than choice due to our goal of conveying choice. We also eliminated themes that seemed too abstract from privacy choice (e.g., leaving or refusing something) or difficult to graphically depict (e.g., third parties). Considering that web icons are generally small, we further eliminated themes that would produce unrecognizable icons when shrunk down in size due to complexity (e.g., exchange/trade-off of data for money). In the end, we identified five themes (see Table 1) that had the potential to represent our three choice-related concepts effectively.

4.1.2 Refinement with graphic designers. Next, we worked with three graphic designers to develop icons for the five themes. The graphic designers worked individually with sketches from our brainstorming sessions as a starting reference, and were encouraged to produce variants and alternative designs, such as varying the shape or size of icon elements. The research team jointly reviewed the graphic designers' work and selected 11 icon designs as candidates for user testing in the icon pre-study.

Table 1 shows all 11 candidate icons. Three icons were intended to convey the broad idea of choice: one featured a toggle - a standard UI element for turning on or off settings [5]; and two featured checkboxes (transitioning from a checked to an unchecked box, or negating a checkbox), since checkboxes are common in online

personal information. The OAG maintains a website that includes documents relevant to CCPA rulemaking [93].

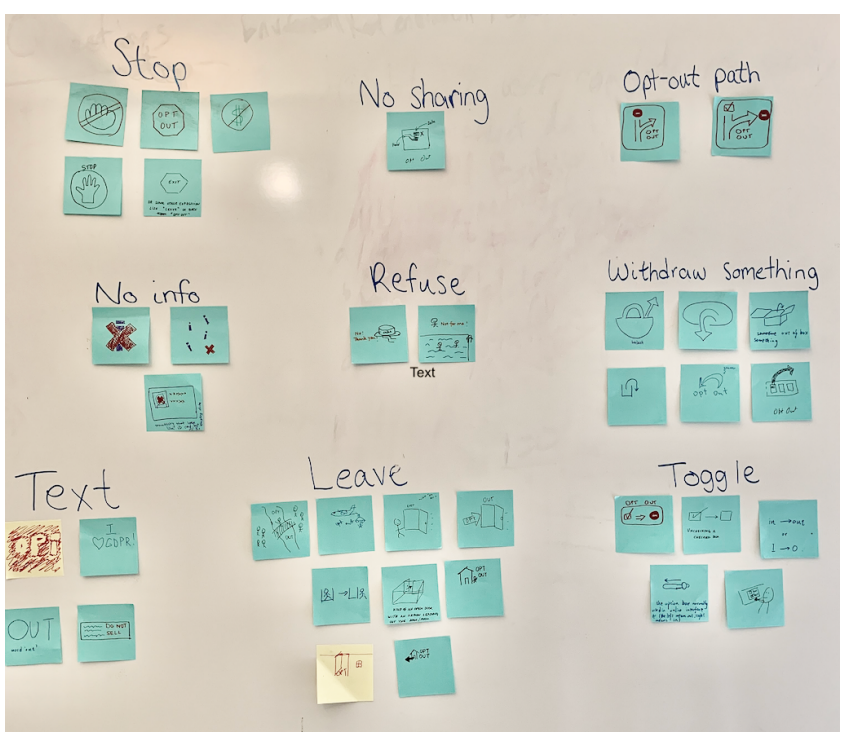

Figure 1: Common themes that emerged in one of the brainstorming sessions for an icon that conveyed opting-out.

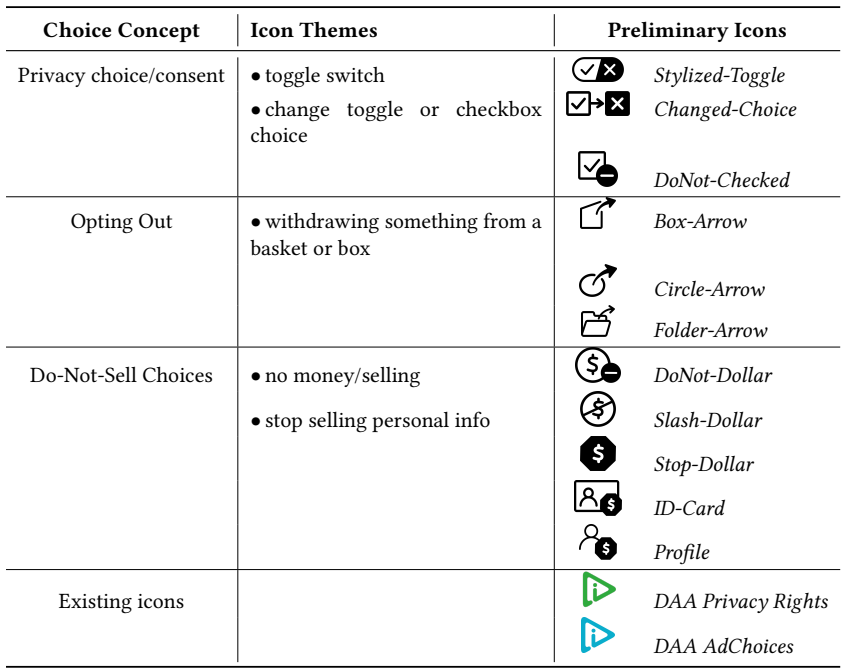

Table 1: Icon themes that emerged in ideation sessions for each choice-related concept, and the corresponding icons included in our preliminary testing.

forms and consent interfaces [5]. Three icons were intended to convey the action of opting out, which is analogous to withdrawing consent: two had an arrow coming out of simple shapes (a circle and a box); and the third used a file folder to represent personal data. Five icons were intended to convey do-not-sell choices: three used different negations of a dollar sign to represent stopping a sale, and two further included a "person" element to represent personal data. To minimize potentially biasing effects of color in our pre-study, we created the initial versions of our icons in black and white. Additionally, we included the DAA's AdChoices [113] and 
Privacy Rights [112] icons in our icon pre-study as a benchmark for industry practices.

\subsection{Preliminary Icon Testing}

We conducted an initial round of user testing on all 11 candidate icons to decide which to test in subsequent studies. We developed an online survey to capture qualitative and quantitative responses that would help us identify feasible icons for indicating the presence of generic privacy choices and do-not-sell choices.

4.2.1 Study protocol. Our initial testing sought to identify difficultto-interpret icons and specific icon elements that help indicate privacy or do-not-sell choices. We implemented a between-subjects design, in which we showed each participant one of the icon candidates at random without context. To examine the impact of placing a link text next to the icon (as required by the CCPA), half of the participants saw the icon displayed with the text "Do Not Sell My Personal Information." We hypothesized this text would aid the comprehension of icons intended to convey do-not-sell choices.

After presenting the icon, we asked participants to provide openended responses regarding their interpretation of the icon and their expectations of what would happen if they clicked on it - this was to capture their unprimed impressions of the icon. As a complementary quantitative data point, we next showed participants all icons, asked them to select which one would best convey the presence of privacy choices and do-not-sell choices respectively, and explain the rationale behind their selection. ${ }^{3}$ We then asked participants about their familiarity and expectations regarding the DAA's AdChoices icon [24] to evaluate the recognizability and comprehension of an already widely deployed privacy choice icon. Lastly, we collected participants' demographic information and asked about awareness of a US law that required companies to provide a "do not sell" option. Appendix A.1 includes the full set of survey questions.

For this and all subsequent studies, we did not collect personal data from participants, and we instructed participants to avoid revealing personal information in their open-ended responses. The Institutional Review Boards at Carnegie Mellon University and the University of Michigan approved all study protocols.

4.2.2 Recruitment and sample demographics. We recruited 240 participants from Amazon's Mechanical Turk (MTurk) to ensure roughly 20 responses per condition - a sufficient number for capturing a variety of opinions for descriptive analysis. We set the recruitment filter as US residents over 18 years old, with a $95 \%$ or higher approval rate. Before answering survey questions, participants reviewed a consent form and confirmed their age and residency eligibility. The average study completion time was 5.25 minutes, and participants were compensated $\$ 1.00$ (average $\$ 11.43 /$ hour).

In line with demographic characteristics of MTurk workers [52], our samples for this and the subsequent studies were diverse but not representative of the US general population: they skewed younger, more male, and more educated. We summarize participant demographics here once as they were fairly uniform across all studies, and provide detailed demographics for each study in Appendix B. Participants were residing in most US states (with 10-20\% living in

${ }^{3}$ The Privacy Rights icon was green when presented alone but black-and-white when presented with other icons to eliminate the impact of color on participants' selection.
California) and somewhat tech-savvy (with $23-48 \%$ reporting education or job experience in computer science, computer engineering, or IT). $3-10 \%$ of participants reported awareness of a US law that required companies to provide a "do not sell" option, with relatively higher percentages in the icon-text combinations and OAG toggle evaluations, indicating a potential increase of awareness after the CCPA went into effect. Once a participant completed one of our studies, we did not permit them to participate in any subsequent studies evaluating icons and link texts.

4.2.3 Data analysis. We conducted a thematic analysis [104] of participants' qualitative responses. One author examined a subset of the qualitative data to identify common themes and developed an initial codebook. The team then discussed the initial codebook, adding and modifying codes as necessary. To ensure high consistency in coding, two authors coded $20 \%$ of all responses and additional responses if needed until reaching a Cohen's $\kappa$ of at least 0.7 , which is considered sufficient agreement [40] (average $\kappa=.81$ across all questions). ${ }^{4}$ Most responses mapped clearly to a code, and ambiguous responses were discussed by multiple researchers before being coded. After we achieved high inter-coder reliability, one researcher coded the remaining responses. We calculated descriptive statistics of coded qualitative data but did not conduct any hypothesis testing, as our primary objective for this pre-study was to eliminate from further consideration icons that appeared confusing or did not effectively convey intended concepts. Eleven responses were excluded from analysis, as they only included text that did not respond at all to the open-ended questions. We note the number of responses excluded from the analysis for this and subsequent studies in Appendix B.

4.2.4 Findings. As shown in Table 2, most icons did not lead to their intended interpretations when shown alone. Participants did not exhibit a clear preference for which icon best represented generic privacy choices, but most chose Slash-Dollar as the icon for representing do-not-sell choices.

A stylized toggle icon best conveyed "choice." Among the three icons that were intended to convey choice, participants commonly associated Stylized-Toggle with the notion of choosing or selecting something. Participants thought of "completion" (i.e., marking something as completed or completed downloads), rather than choice, upon seeing DoNot-Checked. Changed-Choice received a variety of interpretations, suggesting that it would not work well for indicating privacy choices either.

Icons for conveying "opting out" were confusing. Though two participants interpreted Box-Arrow as "removing something" (as intended), other participants interpreted it differently. Participants mostly interpreted Circle-Arrow as something related to motion, and focused on the folder element rather than the arrow in Folder-Arrow; neither prompted participants to think of opting out.

Dollar signs suggested payment rather than selling. All icons intended for do-not-sell choices conveyed a sense of payment or money, but not selling. Interpretations included "cash or American dollars are not accepted," "something is free," "something

\footnotetext{
${ }^{4}$ Responses to the AdChoices interpretation lacked variations, meaning that a single disagreement between coders would cause a significant drop in Cohen's $\kappa$. For this question, we used inter-coder percentage agreement instead to measure inter-coder reliability and ensured the percentage agreement was at least $75 \%$.
} 


\begin{tabular}{|c|c|c|}
\hline Name & Icon & $\begin{array}{l}\text { Common Interpretations (\# of Partici- } \\
\text { pants) }\end{array}$ \\
\hline Stylized-Toggle & & $\begin{array}{l}\text { accept/decline (4); activate/deactivate (2); } \\
\text { true/false (2); mark as completed (1) }\end{array}$ \\
\hline Changed-Choice & & $\begin{array}{l}\text { okay/exit options (1); accept/decline (1); } \\
\text { true/false (1); opposite is true (1); no guesses } \\
\text { (2) }\end{array}$ \\
\hline DoNot-Checked & & $\begin{array}{l}\text { activate/deactivate (2); mark as completed (2); } \\
\text { completed downloads (2); accept/decline (1) }\end{array}$ \\
\hline Box-Arrow & & $\begin{array}{l}\text { removing something (2); okay/exit options } \\
\text { (2); email or message (1); no guesses (1) }\end{array}$ \\
\hline Circle-Arrow & & $\begin{array}{l}\text { move forward/go (3); email or message (1); no } \\
\text { guesses (2) }\end{array}$ \\
\hline Folder-Arrow & & folder/file (4); email or message (3) \\
\hline DoNot-Dollar & & $\begin{array}{l}\text { cancel payment (2); losing money (2); low bal- } \\
\text { ance (2); money/paying ( } 2) \text {; cash/dollars not } \\
\text { accepted (1); something is free or requires no } \\
\text { money (1) }\end{array}$ \\
\hline Slash-Dollar & & $\begin{array}{l}\text { cash/dollars not accepted (4); something is free } \\
\text { or requires no money (3); money/paying (1) }\end{array}$ \\
\hline Stop-Dollar & & $\begin{array}{l}\text { money/paying (4); account balance (2); some- } \\
\text { thing costs money (2); something is free or re- } \\
\text { quires no money (1); cash/dollars not accepted } \\
\text { (1) }\end{array}$ \\
\hline ID-Card & & $\begin{array}{l}\text { payment method (4); something related to } \\
\text { a person and money (3); something costs } \\
\text { money (2); account balance (1); no guesses (1) }\end{array}$ \\
\hline Profile & & $\begin{array}{l}\text { money/paying (2); stop spending money (2); } \\
\text { something costs money (2); }\end{array}$ \\
\hline$D A A$ & $D$ & $\begin{array}{l}\text { more information (3); move forward/go (2); play } \\
\text { button (2) }\end{array}$ \\
\hline
\end{tabular}

Table 2: Participants' coded open-ended responses to "What does this symbol communicate to you?" from conditions in which the icon was shown without a link text in the icon preliminary testing, along with a code's number of occurrences. Interpretations that align with the icon's intended meaning are bolded.

requires payment," and "something related to an account balance." Promisingly, three participants connected ID-Card with a person and money, which aligns with its intended purpose of signaling do-not-sell choices.

No clear preference for the privacy choices icon. Participants were divergent in their opinions of which icon best represented choices about the use of personal information (see Figure 2). Stylized-Toggle was selected most frequently, though ID-Card, DAA, and Folder-Arrow were not far behind. In open-ended responses, participants identified certain icon elements that conveyed privacy choices to them, including "select/choose" (32.3\%), "money/selling" (21.0\%), "personal information" (19.2\%), and "stop/do not" (16.6\%). The mentioning of "money/selling" and "stop/do not" suggests

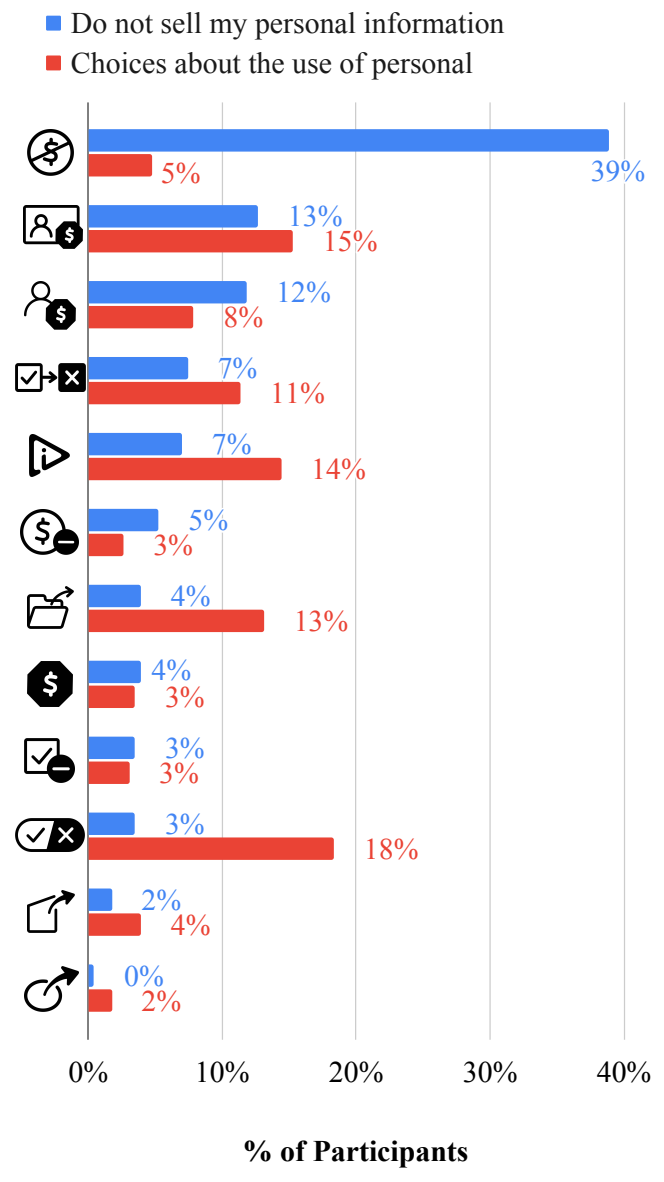

Figure 2: Preliminary testing participants' selections for an icon that best conveys there's an option to (1) "tell websites 'do not sell my personal information"' (blue); and (2) "make choices about the use of my personal information” (red).

potential priming effects from the question that asked about the best icon for do-not-sell choices or the "Do Not Sell My Personal Information" link text when presented.

Slash-Dollar preferred as "do-not-sell” icon. Participants exhibited a clear preference for which icon best represented do-notsell choices as $38.9 \%$ selected Slash-Dollar (see Figure 2). In openended responses, participants mentioned "money/selling” (48.9\%), "stop/do not" (46.7\%) and "personal information" (21.0\%) as important icon elements for conveying do-not-sell choices. Participants preferred "stop/do not" to be represented by a circle with a slash, rather than an octagonal stop sign or a do-not-enter sign, as indicated by the stark difference between Slash-Dollar and DoNotDollar/Stop-Dollar. This suggests that the octagon shape in StopDollar may be difficult to recognize as a stop sign without color, and the "do not enter" sign in DoNot-Dollar was not widely recognized, or was misidentified as a minus sign. 


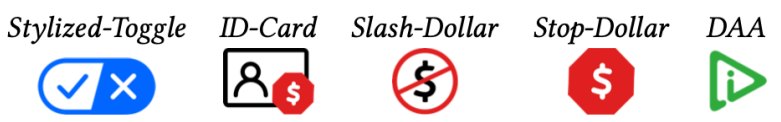

Figure 3: Promising icons from preliminary testing in their refined versions.

\subsection{Refined Icon Testing}

Our preliminary testing suggested comprehension issues with most icons but surfaced some promising candidates. In selecting icons for further testing, we included Stylized-Toggle and ID-Card as candidates for privacy choices: the former appeared to communicate "choice" well, and the latter was ranked highly by participants in preliminary testing. For do-not-sell icon candidates, we included Slash-Dollar due to participants' preferences and Stop-Dollar to explore whether color would increase recognition of the stop sign.

We evaluated refined versions of the four icons mentioned above and the DAA's Privacy Rights icon (see Figure 3) to further narrow down icon selections for the larger-scale icon-text evaluation. Specifically, we colored the stop sign and slash red in ID-Card, StopDollar, and Slash-Dollar, and made the dollar sign in Slash-Dollar more readable. We colored Stylized-Toggle blue - a neutral color that does not convey a particular state, unlike green or red.

4.3.1 Study protocol. We followed the same protocol as before to evaluate the five icons. To mitigate a potential priming effect, we randomized the order of the "best icon" questions for privacy/donot-sell choices. We recruited 280 participants (roughly 28 per condition) to detect a medium effect size (.3) [15] with at least $80 \%$ power for our planned statistical analysis. We aimed for a medium effect size due to the study's exploratory nature and to save the budget for oversampling in the icon-text evaluation. The average study completion time was 4.50 minutes, and each participant received $\$ 1.00$ (average $\$ 13.30 /$ hour).

4.3.2 Data analysis. We followed the same qualitative data analysis approach as before $(\kappa=.79)$. Additionally, we collaboratively categorized the codes used to analyze open-ended responses to "What does this symbol communicate to you?" as correct or incorrect interpretations regarding the icon's intended purpose. We then used these binary labels as the dependent variable of Chisquared tests (or Fisher's exact tests when applicable) to determine whether the overall difference in study conditions were statistically significant. Follow-up pairwise comparisons were adjusted with Holm-Bonferroni corrections.

4.3.3 Findings. Participants interpreted Stylized-Toggle as an indicator of some form of choice, and preferred it over other candidates for conveying generic privacy choices. Consistent with the preliminary testing, participants preferred Slash-Dollar for communicating do-not-sell choices. The CCPA link text's presence made participants more likely to expect an icon to lead to do-not-sell choices.

Stylized-Toggle was interpreted as intended. Table 3 provides common interpretations of each icon when displayed without the CCPA link text. A Fisher's exact test showed significant differences between icons, when presented alone, in generating correct interpretations that align with the icon's intended meaning

\begin{tabular}{c|c|l}
\hline Name & Icon & Common Interpretations (\# of Participants) \\
\hline ID-Card & $\mathbf{2} \$$ & $\begin{array}{l}\text { something costs money (9); sending money to some- } \\
\text { one (5); money/paying (5); something related to a } \\
\text { person and money (3); account balance (3); price } \\
\text { related (2); payment methods accepted by website (2) }\end{array}$ \\
\hline Slash-Dollar & $\$$ & $\begin{array}{l}\text { something is free or requires no money (12); } \\
\text { cash/dollars not accepted (6); money/paying (4); sell- } \\
\text { ing is not allowed (1) }\end{array}$ \\
\hline Stop-Dollar & $\$$ & $\begin{array}{l}\text { money/paying (10); stop spending money (5); some- } \\
\text { thing costs money (4); price related (3); sale/discount } \\
\text { (3); no guesses (3) }\end{array}$ \\
\hline Stylized-Toggle & $\checkmark \times$ & $\begin{array}{l}\text { accept/decline something (11); } \\
\text { vate/deactivate something (4); true/false (4); } \\
\text { okay/exit options (3) }\end{array}$ \\
\hline DAA & $\mathbb{2}$ & $\begin{array}{l}\text { more information (11); play button (7); move for- } \\
\text { ward/go (3); ad related (2) }\end{array}$ \\
\hline
\end{tabular}

Table 3: Participants' coded open-ended responses to "What does this symbol communicate to you?" from conditions in which we showed the icon without a link text in the refined icons study, along with a code's number of occurrences. Interpretations that align with the icon's intended meaning are bolded.

$(p<.001, V=.58)$. Pairwise comparisons found that Stylized-Toggle was more likely to be interpreted correctly compared to other icons (all $p<.001$ ). Open-ended responses suggested that Stylized-Toggle was primarily interpreted as an option to "accept/decline" or "activate/deactivate" something. In contrast, the interpretations of other icons often misaligned with their intended meanings. The DAA's Privacy Rights icon conveyed an option to "get more information" but did not suggest a choice or opt-out. Common interpretations of Slash-Dollar were "something is free or does not require money" or "cash or American dollars were not accepted." ID-Card was mostly interpreted as "something costs money." Stop-Dollar was similarly associated with money, but not selling.

Clear icon preference for privacy choices and do-not-sell choices. As shown in Figure 4, when the icons were colorized, participants exhibited a clear preference for Stylized-Toggle to represent choices about the use of personal information. $16.8 \%$ of participants explicitly stated that a toggle "with a checkmark and an $\mathrm{X}$ in it" nicely conveyed choice. Similar to the preliminary testing, Slash-Dollar was selected most frequently as the icon for conveying do-not-sell choices; ID-Card ranked second (see Figure 4).

CCPA link text led to expectations of do-not-sell choices. A Chi-squared test showed that participants who saw the CCPA link text were significantly more likely to interpret the icon as its intended meaning $(p<.001, \phi=.38)$. Of the 139 participants who saw an icon with the CCPA link text, $43.2 \%$ (60) expected some form of choice to stop websites from selling their personal information. $13.7 \%$ (19) expected the ability to configure the types of personal information they could prevent from being sold or entities to which information is sold. $31.7 \%$ (44) expected being immediately opted out of the sale of personal information after clicking. There was no significant difference between icons in creating any of these expectations, suggesting that the link text impacted participants' 


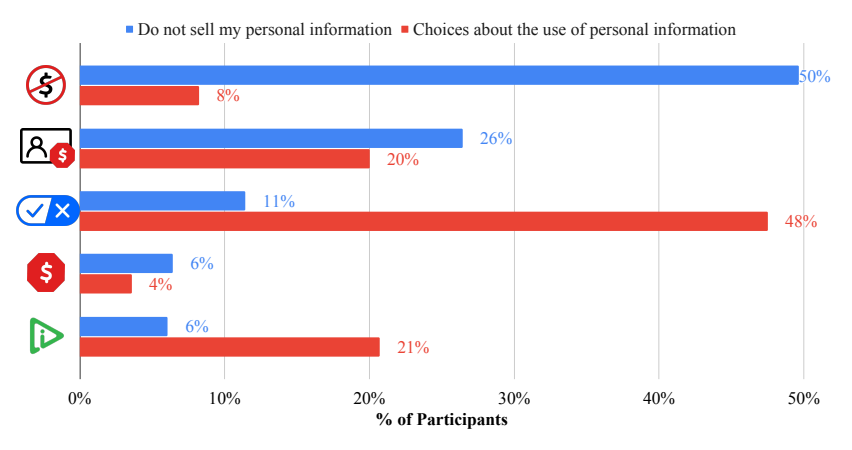

Figure 4: Refined testing participants' selections for an icon that best conveys that there's an option to "tell websites 'do not sell my personal information"' (blue); and "make choices about the use of my personal information" (red).

expectations rather than the icon. Notably, the CCPA link text's presence did not eliminate misconceptions, such as expecting a different type of privacy choice (e.g., opting out of data collection on the website) or interpreting the link text as a warning not to give out their personal information to websites.

DAA's AdChoices icon still mostly unknown. Even though the DAA launched its AdChoices icon in 2010, only 40 (14.3\%) participants recalled seeing this icon before. The most common expectation of the AdChoices icon was that it provided more information about something, as indicated by 152 (54.3\%) participants. Only six participants expected it would lead them to choices related to targeted advertising. Our results confirm Leon et al.'s 2011 findings that there is little recognition of the AdChoices icon [71] - time and widespread adoption does not seem to have increased consumer awareness of this icon.

\section{LINK TEXT PRE-STUDY}

We developed and iteratively evaluated potential link texts to accompany our icons and aid comprehension. "Privacy Choices" emerged as the best candidate for conveying generic privacy controls with few misconceptions, closely followed by "Privacy Options." The CCPA link text variants performed well in conveying do-not-sell opt-outs but did not generalize to other types of privacy controls.

\subsection{Link Text Development}

We generated link text candidates by identifying words or phrases corresponding to the three icon concepts we focused on (choice, opting-out, and do-not-sell). During our ideation, we observed that link texts could follow a pattern of two components: a privacyfocused prefix and, optionally, a choice-focused suffix. We wanted to explore whether the general prefix "privacy" or the more specific prefix "personal info" would more clearly convey the type of choices. For the suffix, we hypothesized that the broad terms "choices" and "options" would create different expectations compared to "opt-out," a more specific type of choice. We also included the two CCPA do-not-sell opt-out texts [88] and their variants - including an abbreviated version ("Don't Sell My Info"), and versions emphasizing choice rather than information (e.g., "Do-Not-Sell Choices") - to

\begin{tabular}{|c|c|}
\hline - Do Not Sell My Personal Information & - Privacy Options \\
\hline - Do Not Sell My Info & - Privacy Opt-Outs \\
\hline • Don’t Sell My Info & - Privacy Choices \\
\hline - Do Not Sell ${ }^{\dagger}$ & - Personal Info Choices \\
\hline - Don’t Sell ${ }^{\dagger}$ & - Personal Info Options \\
\hline - Do-Not-Sell Choices ${ }^{\dagger}$ & - Personal Info Opt-Outs \\
\hline • Do-Not-Sell Options & - Do Not Sell My Info Choices \\
\hline - Do-Not-Sell Opt-Outs ${ }^{\dagger}$ & - Do Not Sell My Info Options \\
\hline
\end{tabular}

Table 4: Link texts tested in the link text pre-study.

control for confounds and explore potential alternatives to the CCPA link texts.

Our initial set included 14 link texts revolving around six words or phrases: personal info/privacy/do-not-sell for the prefix, and choices/options/opt-outs for the suffix. After preliminary testing, we eliminated four with poor comprehension and added two for further testing. Table 4 shows the full set of link texts we evaluated.

\subsection{Preliminary Link Text Testing}

We tested the initial link text set using a similar protocol as the icon pre-study. Based on the findings, we eliminated four candidates from subsequent testing and added two more variants of the CCPA link texts.

5.2.1 Study protocol. We showed each participant one of the 14 candidate link texts at random, styled as a hypertext link but nonclickable, without an icon or other context. We asked participants to describe their expectations of what would happen if they clicked on the link and interpretations of specific text components. Then, we presented eight scenarios constructed from open-ended responses from the icon pre-study and asked participants to rate the likelihood that clicking on the link would lead to each scenario. Two scenarios were accurate expectations related to privacy notices and choices, three were accurate expectations related to do-not-sell, and three were misconceptions (see Q3 in Appendix A.2). Lastly, participants were asked demographic questions and about their familiarity with the CCPA. We recruited 140 participants on MTurk (roughly ten responses per condition) to have a diverse set of qualitative responses for descriptive analysis. The average study completion time was 4.20 minutes, and each participant received $\$ 1.00$ (average $\$ 14.29 /$ hour).

5.2.2 Data analysis. We coded participants' open-ended responses using the same thematic analysis approach as in the icon pre-study $(\kappa=.89)$. The coded data was used for descriptive analysis only, as our primary goal was to identify link texts with high rates of misconceptions and eliminate them from further consideration.

5.2.3 Findings. Our preliminary testing of link texts suggested a greater influence of the prefix, rather than the suffix, on expectations of what happens after clicking the link. "Personal information" was understood as personally-identifiable information, and its absence led to misconceptions about the word "sell." 
"Personal information" was primarily interpreted as PII (personally identifiable information). When asked to interpret the phrase "personal information," "personal info," or "info," 33 of the 57 participants (57.9\%) who saw a corresponding link text listed examples of PII, such as name and birthday. 11 participants interpreted the phrase as demographic information, such as age or gender. Nine participants thought it referred to their IP address or location, and another nine believed it referred to cookies or past activities on the website or elsewhere.

"Sell" on its own was often misunderstood. Without an explicit reference to personal information, participants struggled to identify the subject to which "sell" referred. Among the 45 participants who saw one of the "do not sell" variants without "personal information" or "my info," 18 (40.0\%) thought the sale referred to a physical product. Four thought the sale was related to stocks or money, and five did not know what the sale is about. Given that participants saw the link text with no further context, it is not surprising that such misconceptions occurred.

\subsection{Refined Link Text Testing}

Our preliminary testing showed that link texts containing the word "sell" without "info" did not convey privacy choices or do-not-sell choices well. Therefore, we eliminated four corresponding link texts from further testing but retained "Do-Not-Sell Options," which conveyed a control/choice related to personal information about as frequently as "Privacy Opt-Outs" and "Personal Info Options." We added two new link texts ("Do Not Sell My Info Choices" and "Do Not Sell My Info Options") to assess how adding choice-related suffixes would affect the interpretation of the CCPA-mandated link texts. We did not test "Do Not Sell My Info Opt-Outs," as our preliminary testing suggested "opt-outs" might be less intuitive than "choices" or "options."

5.3.1 Study Protocol. We recruited 400 additional participants, roughly 33 per condition, to detect a medium effect size (.3) with at least $80 \%$ power for our planned statistical analysis comparing expectations generated by the candidate link texts. The average study completion time was 4.1 minutes, and participants were compensated $\$ 1.00$ (average $\$ 14.63 /$ hour). Since we used the same protocol and survey instrument, we aggregated participant responses with those collected from the preliminary testing for the analysis.

5.3.2 Data Analysis. We followed the same qualitative data analysis approach as in previous studies; two authors coded $20 \%$ of the data $(\kappa=.81)$ and one author coded the remainder. For this and the following studies, we structured the codebook hierarchically by grouping codes into four categories (high-level codes) for categorylevel analysis. Specifically, we labeled "yes" or "no" for whether a code conveyed (1) the concept of choice; (2) the ability to opt out of the sale of personal information; (3) the concept of privacy broadly; and (4) misconceptions. ${ }^{5}$ Three authors completed the mapping for all codes together and resolved any disagreements. We then used the values of these categorizations as the dependent variables in Pearson chi-square or Fisher's exact tests, with link

\footnotetext{
${ }^{5}$ For example, the response "It would give you the option to not have your personal information given, shared, or sold to someone else" was coded as "choices: do not sell." For high-level categories, the code was labeled as "yes" for conveying choice and do-not-sell, and "no" for conveying privacy or a misconception.
}

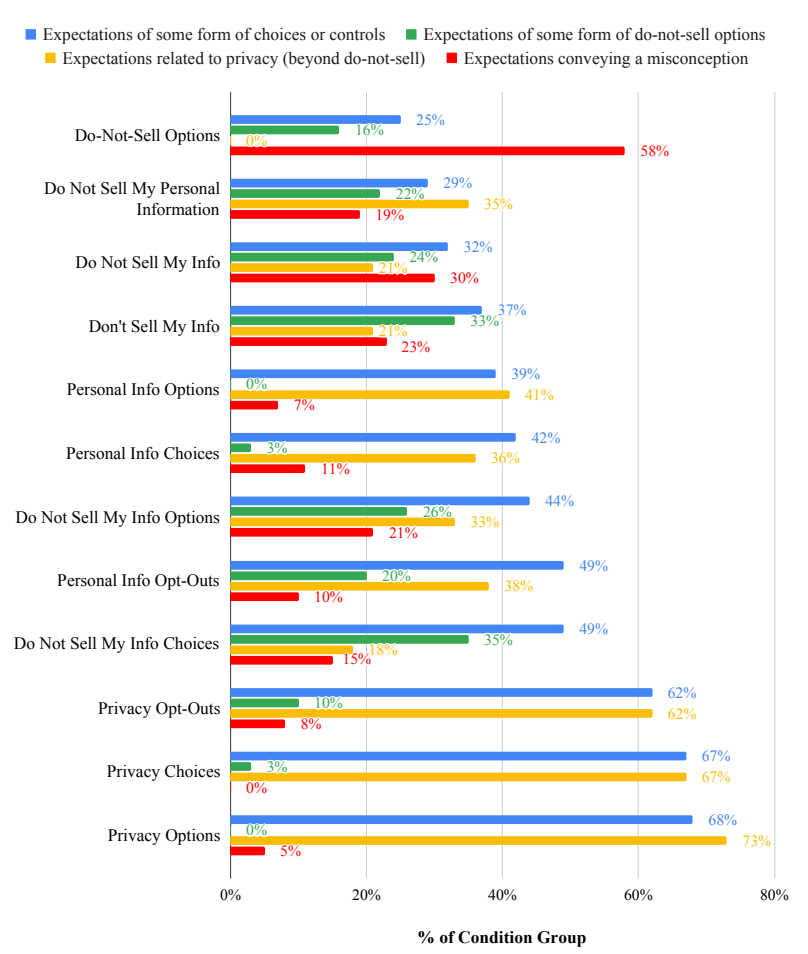

Figure 5: Distribution of expectations in response to "What do you think would happen if you clicked on this [link]?" in our link text pre-study.

text conditions as the independent variable. Pairwise comparisons were Holm-Bonferroni corrected.

5.3.3 Findings. As seen in Figure 5, participants' expectations significantly varied across link texts. "Privacy Choices" created the least misconceptions. The CCPA link texts and their variants successfully led to expectations of do-not-sell choices.

Link text suffix did not impact expectations of choices. $47.9 \%$ of participants expected to see some form of choices, including those related to privacy and do-not-sell. As seen in Figure 5, there was a significant overall difference between conditions $(p<.001$, $V=.27)$. Pairwise comparisons revealed that the only significant difference was between "Privacy Options" and "Do-Not-Sell Options" ( $p=.04) ; 67.6 \%$ and $25.0 \%$ of participants in those conditions expressed expectations of choices, respectively. The choice-related suffixes (i.e., "choices," "options," or "opt-outs") did not appear to impact participant expectations of choices, given the small differences between link texts with the same privacy-related prefix.

CCPA link text variants led to expectations of do-not-sell choices but did not generalize. As seen in Figure 5, there was a significant difference between conditions in generating expectations of do-not-sell choices $(p<.001, V=.34)$, or something more broadly related to privacy $(p<.001, V=.42)$. Link texts beginning with "Do Not Sell" most often led to expectations of 
do-not-sell choices, with "Do Not Sell My Info Choices" performing significantly better than "Personal Info Options" ( $p=.005)$, "Privacy Options" ( $p=.008)$, and "Privacy Choices" $(p=.04)$ in this regard. $35.0 \%$ of participants who saw "Do Not Sell My Info Choices" expected do-not-sell choices, whereas no participants who saw "Personal Info Options" or "Privacy Options" expressed the same expectation. However, link texts beginning with "Do Not Sell" did not effectively convey broader privacy-related information or options. "Privacy Options," "Privacy Choices," and "Privacy Opt-Outs" were all significantly better than "Do-Not-Sell Options" (all $p<.001)$, "Do Not Sell My Info Choices" (.0003<p<.012), "Don't Sell My Info" (.001 $<p<.04)$, and "Do Not Sell My Info" $(.002<p<.05)$ for this purpose. $67.1 \%$ of participants who saw a "Privacy" prefixed link text described a privacy-related expectation, compared to $21.4 \%$ who saw a "Do Not Sell" prefixed link text.

"Privacy Choices" generated the least misconceptions. As seen in Figure 5, the distribution of misconceptions were not even across conditions $(p<.001, V=.39)$. Pairwise comparisons revealed that "Privacy Choices" created significantly fewer misconceptions than "Do Not Sell My Info" $(p=.04)$. Among the 63 participants who saw one of the link texts beginning with "Do Not Sell," some thought the link would lead to phishing/malware risks (16), investment advice (8), the site's policy on selling items (8), and ads for privacy products or other services (6).

Some link texts might apply to both privacy choices and do-not-sell choices. In examining participants' Likert responses to the predefined scenarios, five link texts were rated as "definitely" or "probably" likely to lead to choices about how personal information is used and shared by over three quarters of participants. Among them, "Personal info Choices," "Privacy Opt-Outs," "Do Not Sell My info Options," and "Privacy Options" were also among the top five link texts rated as "definitely" or "probably" likely to lead to the scenario describing choices about the sale of personal information. This suggests that these four link texts had the potential to convey both generic privacy choices and do-not-sell choices relatively well.

\section{ICON-TEXT COMBINATIONS EVALUATION}

Our pre-studies suggested a need for combining icons with link texts, consistent with prior research and recommendations [38, 122]. Icons alone do not necessarily translate to correct expectations even with a certain degree of familiarity $[58,102]$, as reflected by our findings on the DAA's AdChoices icon. Similarly, link text alone might not stand out. Pairing the two together can attract user attention and aid comprehension [51]. We conducted a large-scale evaluation to find icon-text combinations that accurately convey privacy choices and do-not-sell choices.

\subsection{Method}

For icons, we selected Stylized-Toggle and Slash-Dollar, since they were the most preferred for indicating privacy choices and do-notsell choices respectively. We also included DAA's Privacy Rights icon because of its potential for widespread adoption by DAA member companies. For link texts, we selected "Privacy Options" and "Privacy Choices" since they best generated expectations of choices/controls and expectations related to privacy (see Figure 5).
We also included the two CCPA-mandated link texts since they conveyed do-not-sell choices well. We did not include any variants of the CCPA link texts since the choice-related suffix did not influence participant expectations. Additionally, we included "Personal Info Choices" since Likert responses to predefined scenarios suggested it worked well to communicate both do-not-sell choices and broader privacy controls.

6.1.1 Study protocol. To measure to what extent icons and link texts interact with each other in shaping participant expectations, we used a nearly full-factorial experimental design including four icon conditions and six link text conditions (a total of 23 conditions). The four icon conditions were the DAA's Privacy Rights icon, SlashDollar, Stylized-Toggle, and no icon. The six link text conditions were "Do Not Sell My Personal Information," "Do Not Sell My Info," "Privacy Choices," "Privacy Options," "Personal Info Choices," and no link text. We excluded the combination of no icon and no link text since participants would not see any information. Our examination of icon-text combinations was exploratory - even though the prestudies indicated that some icons and link texts perform better than others for certain purposes, interaction effects might exist between the icon and text, making it difficult to generate specific hypotheses.

We followed a between-subjects design, showing each participant an icon-text combination at random. While we presented icons and link texts with no context in the pre-studies, here we showed the icon and link text together on a fictitious online shoe retailer website (see Figure 6) to emulate how consumers might encounter them in the wild. We modified the eight scenarios for Likert questions based on common expectations uncovered in the link text pre-study; two were correct expectations, two were semi-correct expectations, and the rest were misconceptions about unwanted outcomes (see Q3 in Appendix A.3). We recruited 1,468 MTurk participants (roughly 64 per condition) based on heuristics that would allow us to run planned regressions [96]. The average study completion time was 4.55 minutes, and participants were compensated $\$ 1.00$ (average \$13.19/hour).

6.1.2 Data analysis. We followed the same qualitative analysis approach as in the link text pre-study $(\kappa=.83)$ before using the data for quantification. ${ }^{6}$ We coded participants' responses about expectations to identify common themes, then categorized individual codes based on whether they convey the idea of choice, do-not-sell choices, privacy broadly, or misconceptions. We then ran logistic regressions using these high-level code categories as the dependent variable, the icon-text combination condition as the main independent variable, and participant demographics as control independent variables. We ran additional logistic regressions with the same independent variables on a binary variable that represented participants' expected likelihood of each predefined scenario. ${ }^{7}$ We applied Holm-Bonferroni corrections to $p$-values in all regressions since we conducted multiple tests without preplanned hypotheses [4]. Detailed regression results are provided in Tables 6 and 7 as part of Appendix C.1.

\footnotetext{
${ }^{6}$ There was little diversity in responses to the question regarding the meaning of "sell" in the link text. Thus, we used percentage agreement rather than Cohen's $\kappa$ to measure inter-coder reliability and ensured the percentage agreement was at least $75 \%$.

7 "Definitely" and "probably" were coded as "expected" (expecting the scenario would happen) and the other answer options were coded as "unexpected."
} 


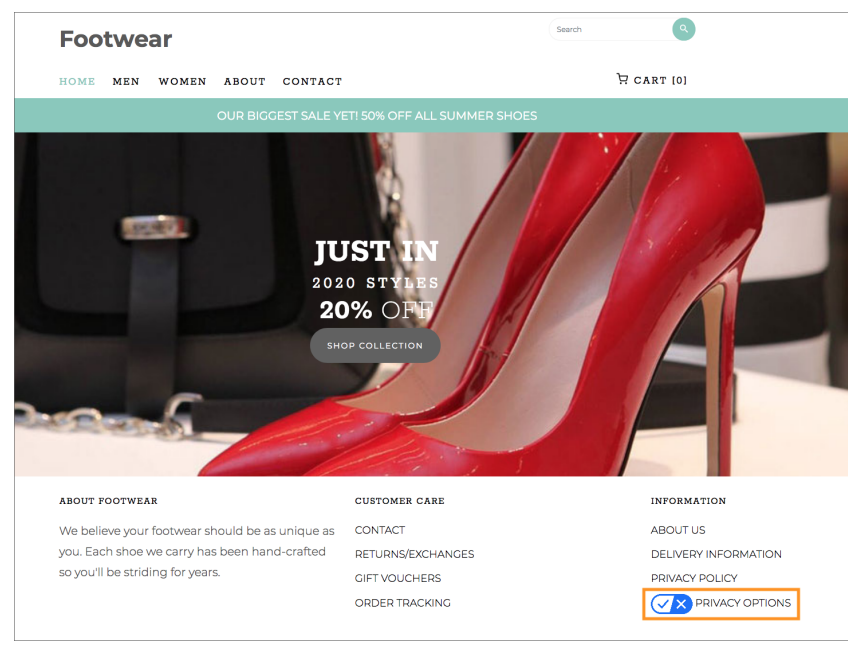

Figure 6: Icon and link text presented on a fictitious online shoe retailer webpage used in the icon-text combination evaluation. The icon and link text were highlighted with an orange rectangle to attract participants' attention. Shown is the condition combining Stylized-Toggle (icon) and "Privacy Options" (link text).

\subsection{Findings}

We found significant differences between icon-text conditions in creating expectations of privacy choices or do-not-sell choices; link texts impacted participant expectations more than icons in this regard. Furthermore, Slash-Dollar and "Personal Info Choices" generated more misconceptions than the other icons or link texts.

Conveying privacy choices. Regressions of participants' categorized open-ended expectations (Table 6 in Appendix C.1) compared how well different icon-text combinations conveyed the concepts of choice (e.g., "My choices would pop up on the screen") and privacy (e.g., "It will enable a more private experience"). Compared to Toggle-Privacy Options as the baseline, combinations including the "Privacy Options" or "Privacy Choices" link text, as well as Stylized-Toggle by itself, performed similarly in generating privacy-related expectations; participants in all other combinations were significantly less likely to expect something related to privacy $(.005<O R<.13$, all $p<.001)$. Furthermore, participants were significantly less likely to expect some form of choice when seeing the link text "Personal Info Choices" without Stylized-Toggle, or $D A A /$ Dollar without an accompanying link text $(.03<O R<.27$, $.001<p<.03)$.

Figure 7a shows participants' Likert responses to the generic privacy choice scenario. Overall, Toggle-Privacy Options was the best candidate for conveying "choices about how personal information is used or shared": $93.4 \%$ of participants who saw this combination thought they would definitely or probably be led to privacy choices. Regressions of Likert responses (Table 7 in Appendix C.1) further showed that participants were significantly more likely to expect privacy choices when seeing Toggle-Privacy Options, compared to Toggle-Do Not Sell My Personal Information, Slash-Dollar icon alone, and $D A A$ icon alone $(.03<O R<.17, .001<p<.009)$.

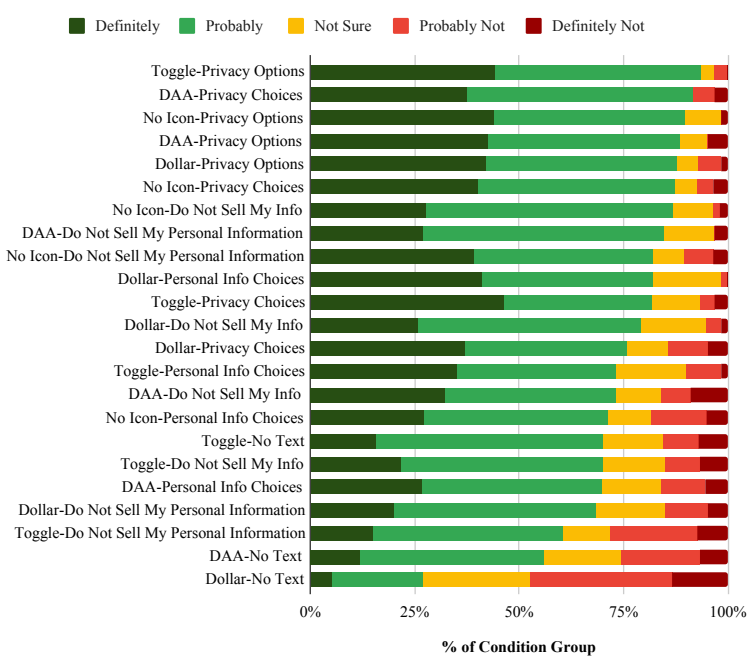

(a) "It [the symbol/phrase] will take me to a page with choices about how my personal information is used and shared by the website."

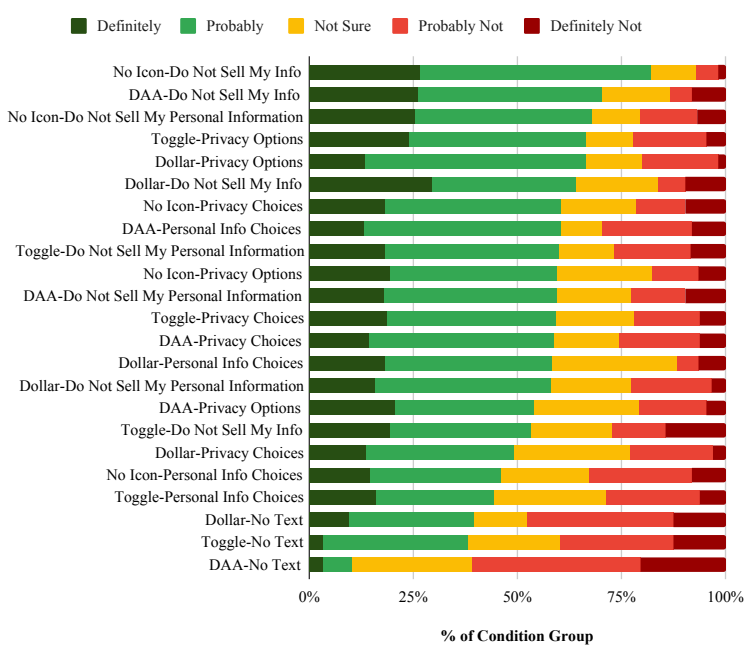

(b) "It [the symbol/phrase] will take me to a page with choices about the sale of my personal information."

Figure 7: Distribution of Likert responses across conditions in icon-text combinations evaluation.

However, the differences between Toggle-Privacy Options and other conditions with "Privacy Options" as the link text were minimal and not significant in regressions. Most combinations involving the "Privacy Options" and "Privacy Choices" link texts effectively conveyed privacy choices.

Conveying do-not-sell choices. Regressions of participants' categorized open-ended expectations indicated that the two CCPAmandated link texts significantly outperformed other link texts in creating the expectation of do-not-sell choices (e.g.,"It would let you opt out of them selling your information"). Relative to "Do Not Sell My Personal Information" with no icon, all conditions with the 
link texts "Privacy Options," "Personal Info Choices," and "Privacy Choices" performed significantly worse in generating expectations of do-not-sell choices $(.01<O R<.13$, all $p<=.001)$. There were no significant differences between "Do Not Sell My Personal Information" or "Do Not Sell My Info" in this regard.

Figure 7b shows participants' Likert responses to the do-not-sell choices scenario. The three conditions with the highest percentage of definitely/probably responses all included one of the CCPA link texts: No Icon-Do Not Sell My Info (82.1\%), DAA-Do Not Sell My Info (70.5\%), and No Icon-Do Not Sell My Personal Information (67.8\%). Regressions on Likert responses further showed that No Icon-Do Not Sell My Personal Information performed significantly better than the $D A A(O R=.06, p<.001)$ and Slash-Dollar icons alone $(O R=.28$, $p=.04)$ in conveying do-not-sell choices, suggesting effectiveness of the CCPA link texts in this regard.

Stylized-Toggle was occasionally perceived as an actual control button. While Toggle-Privacy Options conveyed privacy choices well and the two CCPA mandated link texts conveyed do-not-sell choices well, putting Stylized-Toggle next to the CCPA link texts led to an unintended consequence. $40.0 \%$ of participants who saw Toggle-Do Not Sell My Personal Information expected that clicking on them would definitely or probably "give the website permission to sell my personal information." Stylized-Toggle significantly increased the likelihood of this misconception compared to no icon $(O R=5.25, p=.02)$ when combined with the "Do Not Sell My Personal Information" link text. This suggests that participants might perceive Stylized-Toggle as an actual control switch for the sale of one's personal information on the website when the icon was next to the CCPA link texts. However, we did not observe a similar pattern in participants' open-ended expectations - this expectation only emerged when we explicitly asked participants whether clicking the icon would give the website permission to sell their personal information, indicating a potential priming effect.

Misconceptions with Slash-Dollar icon and "Personal Info Choices.” Regressions of participants' categorized openended expectations revealed that Slash-Dollar without a link text significantly increased the likelihood of misconceptions relative to Toggle-Privacy Options $(O R=67.2, p<.001)$. Among the 371 participants who saw Slash-Dollar, 33 (8.9\%) expressed expectations of payment options, particularly related to secure or encrypted payment (e.g., "It would present your rights to pay through secure links"). These findings indicate that the Slash-Dollar icon, even when paired with a link text, might be too suggestive of payment, transaction, or other financial concepts that do not concern personal information.

Also relative to Toggle-Privacy Options, all conditions with "Personal Info Choices" increased the likelihood of misconceptions $(11.9<O R<18.1, .005<p<.04)$. Only $42.0 \%$ of participants who saw "Personal Info Choices" accurately interpreted choices as controls related to the collection, processing, and sharing of their personal data or broader privacy choices, compared to $66.5 \%$ of those who saw "Privacy Choices." Misinterpretations of choices most frequently included profile settings related to purchasing shoes (16.7\%; e.g., "Probably it would let you input your shoe size, height, favorite styles, etc. for a more customized look"). Other misconceptions included that the link would lead to choices about shoe styles or sizes available on the website (13.1\%) and choices related

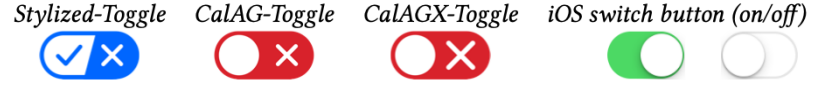

Figure 8: Our stylized toggle, OAG's proposed opt-out button, its variant, and the iOS switch button.

to payment methods (1.6\%). The remaining participants were either not sure about or did not specify the types of choices they expected.

\section{OAG ICON EVALUATION}

In February 2020, the California Attorney General's office (OAG) released the first set of modifications to the CCPA regulations [91] after we had shared our results with them. The proposed modifications included an opt-out icon (CalAG-Toggle) that was similar, but not identical to our Stylized-Toggle icon (see Figure 8).

Our icon-text combinations evaluation suggested that StylizedToggle might occasionally be perceived as an actual control switch rather than an icon when paired with the CCPA-mandated link texts. We were concerned that CalAG-Toggle would make this misconception even more likely for two reasons. First, CalAG-Toggle closely resembled the toggle switch in iOS (see Figure 8). By contrast, Stylized-Toggle used a checkmark and "X" to visually convey the availability of options and a dividing line to differentiate it from a real toggle control. Second, CalAG-Toggle being in red created a potentially confusing double negative when paired with "Do No Sell My Personal Information." One could interpret it as either "my data is currently being sold" (because red indicates the setting "Do Not Sell My Personal Information" being off), or "my data is currently not being sold" (because red indicates the sale of personal information is prohibited). In contrast, Stylized-Toggle used blue, a neutral color that does not convey a particular state. We conducted a follow-up study to examine whether the style and color of CalAG-Toggle might diminish icon comprehension compared to Stylized-Toggle.

\subsection{Method}

We used the method already employed in our icon-text combinations evaluation to test the OAG's proposed icon.

7.1.1 Study protocol. To understand to what extent icon style and color jointly shape participant interpretations, we implemented a full factorial design that included two color conditions (red, blue) and three style conditions (six conditions total). In addition to Stylized-Toggle and CalAG-Toggle, we created a third style condition, CalAGX-Toggle (see Figure 8), which seeks to improve the visual aesthetics of CalAG-Toggle by enlarging the " $\mathrm{X}$ " to make it visually equivalent to the circle.

As before, we used a between-subjects design, showing participants one of the six icons at random next to "Do Not Sell My Personal Information" on a fictitious online shoe retailer website. In addition to their open-ended expectations, we asked participants about the likelihood of eight scenarios occurring on a Likert scale. In order to understand whether participants viewed the toggle as an actual control switch, we included two misconception scenarios of immediate settings changes (see Q3 in Appendix A.4). We recruited 


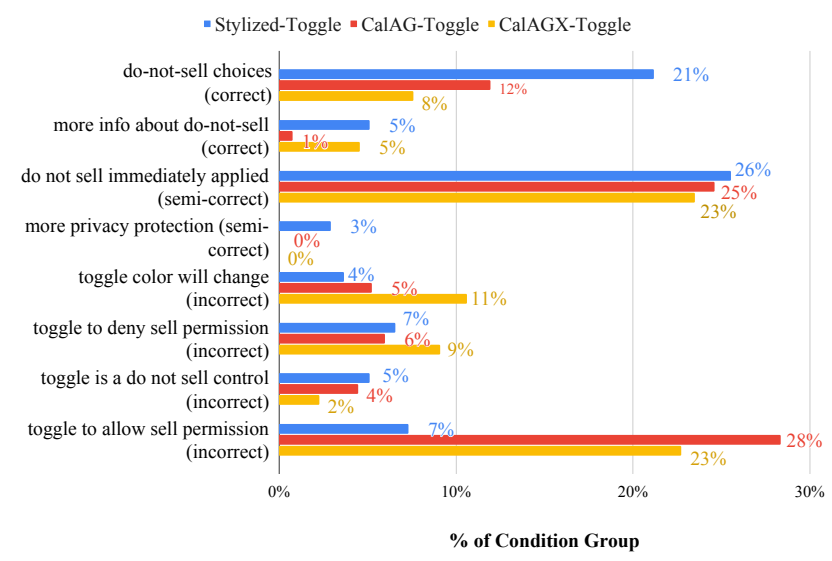

Figure 9: Common expectations of what would happen after clicking based on open-ended responses in conditions with Stylized-Toggle $(n=137)$, CalAG-Toggle $(n=134)$ and CalAGXToggle $(n=132)$.

421 MTurk participants (roughly 70 per condition) for this study based on heuristics for running our planned regressions [96]. The average study completion time was 4.6 minutes, and participants were compensated $\$ 1.00$ (average $\$ 13.04 /$ hour).

7.1.2 Data analysis. We used the same approach employed in our previous studies to analyze qualitative data $(\kappa=.90)$. Additionally, we grouped codes into high-level categories as to whether the code conveyed (1) any misconceptions or (2) the icon was perceived as an actual control switch. We then ran logistic regressions on these coded expectations and Likert responses (converted into a binary variable) to scenarios. We treated the interaction term [17] between icon color and style as the key independent variable, and participant demographics as the control independent variables. ${ }^{8}$ Detailed regression results are provided in Tables 8 and 9 of Appendix C.2. We did not apply corrections to $p$-values since we ran a small number (2) of regressions with preplanned hypotheses (i.e., Stylized-Toggle would perform better than CalAG/CalAGX-Toggles) [4].

\subsection{Findings}

We found that Stylized-Toggle better conveyed do-not-sell choices than the OAG's proposed opt-out icon and its variant with fewer toggle-related misconceptions. The icon's color (red or blue) did not significantly alter participant expectations in most cases.

Stylized-Toggle better created expectations of do-not-sell choices. Figure 9 shows expectations of what would happen after clicking an icon. The most frequent expectation regarding StylizedToggle $(29,21.2 \%)$ was to be directed to a page with choices about the sale of personal information, a correct and desired interpretation according to the CCPA [88]. This expectation, however, was

\footnotetext{
${ }^{8}$ Following statistical analysis guidelines [103], for any model in which the interaction effect between style and color was not significant, we compared its performance with another model without the interaction term (i.e., style and color was examined in isolation as main effects). If the "interaction model" provided a much better fit to the data than the "main effect only model," we report results from the first model; otherwise, we report results from the latter model.
}

mentioned much less often in conditions involving CalAG-Toggle $(16,11.9 \%)$ and CalAGX-Toggle (10, 7.6\%). The significant differences were confirmed by regressions on Likert responses to the do-notsell choices scenario, in which participants who saw Stylized-Toggle were significantly more likely to expect "it will lead me to a page where I can choose whether or not the website can sell my personal information" compared to CalAG-Toggle $(O R=.40, p<.001)$ and CalAGX-Toggle $(O R=.41, p=.001)$.

Stylized-Toggle led to fewer toggle-related misconceptions. Regressions on participants' categorized open-ended expectations revealed that CalAG-Toggle and CalAGX-Toggle were significantly more likely to generate misconceptions compared to Stylized-Toggle $(O R=2.3, O R=2.4$; both $p=.003)$. Examples of these misconceptions include perceiving the toggle icon as an actual switch, expecting a negative outcome (e.g., more tracking), or believing that nothing would happen. Specifically, participants who saw CalAG-Toggle and CalAGX-Toggle were significantly more likely to perceive the toggle as an actual control switch compared to Stylized-Toggle $(O R=2.4, p=.003 ; O R=2.4, p=.004)$. A participant quote that conveyed this misconception is "It would change between red and green depending on if I wanted to allow it."

As shown in Figure 9, the most frequent expectation in conditions involving CalAG-Toggle $(38,28.4 \%)$ and CalAGX-Toggle (30, 22.7\%) was that the icon was an actual toggle switch currently set to "Do Not Sell My Personal Information" - clicking would give the website permission to sell the user's personal information, which is the opposite of the intended meaning. Users who have this notion might avoid clicking the icon or link text for fear of losing their privacy and thus lose the opportunity to exercise the do-not-sell optout. In contrast, only 10 (7.3\%) participants who saw Stylized-Toggle mentioned this misconception.

Another misconception that occurred for all three icon styles (9, $6.6 \%$ for Stylized-Toggle; 8, 6.0\% for CalAG-Toggle and 12, 9.1\% for CalAGX-Toggle) was that the website is currently selling the user's personal information, and that clicking the toggle would stop it. Participants who held this misconception understood the icon's purpose but misinterpreted the icon's functionality - according to the CCPA [88], the icon should take users to respective settings but is unlikely to result in immediate changes. Regressions on the Likert responses for the respective scenario revealed interaction effects between toggle style and color; Stylized-Toggle in blue significantly decreased the likelihood of this misconception compared to StylizedToggle in red $(O R=2.78, p=.006)$ and CalAGX-Toggle in blue $(O R=$ $2.75, p=.009)$. This misconception is not particularly problematic as it is less likely to discourage users from clicking. However, a privacy choice icon ideally should communicate both its intention and its function accurately.

\section{DISCUSSION}

Our findings provide insights into the design and effectiveness of icons and link text in conveying privacy choices. Below we discuss our study's limitations and outline implications for design practice and privacy regulations. 


\subsection{Limitations}

Our research has several limitations. First, we recruited all participants from Mechanical Turk, and they were more educated and tech-savvy than the U.S. general population. Nonetheless, prior work has shown that MTurkers are more demographically diverse than student samples $[10,13]$ and that they offer similar responses to security and privacy surveys as traditional participant pools [99] Second, our experiments focused on one application scenario (a fictitious online shoe retailer), which might have primed participants (e.g., to associate the dollar sign with payment and "sell" with shoe discounts). That noted, participants' responses for our best performing icons/link texts did not indicate that the website context affected their interpretations. Third, we measured the perception and comprehension of the icon/text by presenting them in a static screenshot; we did not measure whether participants would notice the icon/text on their own or how participants would interact with the provided choices as that was not the focus of this study. ${ }^{9}$ Fourth, we did not investigate accessibility issues or evaluate the use of icons with screen readers. Lastly, we did not directly compare our privacy choice icons with icons focusing on different privacy-related aspects (e.g., those that seek to visualize the concept of privacy itself or specific data practices [102]), which could be a contribution of future work.

\subsection{Design Implications}

Icons for privacy choices should be rooted in simple and familiar concepts. Stylized-Toggle was participants' favorite privacy choice icon in the pre-study, and performed best in conveying privacy choices when paired with "Privacy Options" in the icontext combinations evaluation. Stylized-Toggle adopts a minimalistic design and conveys the notion of choice using a toggle - a familiar and common UI element representing the ability to make selections [5]. Nonetheless, the OAG icon evaluation shows the importance of an icon taking inspirations from rather than copying other familiar UI elements to convey the intended concept without creating confusion. Conversely, the icons that were comprehended poorly and thus excluded after the icon pre-study either attempted to convey a more abstract concept (e.g., the three icons that intended to convey "opt out") or appeared too complicated as they combined multiple concepts (e.g., ID-Card and Profile combined elements representing "do not," "personal information," and "money/selling").

Our findings suggest that an icon for privacy choices should focus on a simple and familiar concept, like choice, instead of abstract or complex concepts. For the same reason, we hypothesize that a choice-focused icon would work better than an icon attempting to convey "privacy" in indicating privacy choices future work is needed to validate this hypothesis, as we did not test privacy-focused icons. While prior work has proposed graphical representations of privacy - such as sunglasses, keyholes, locks, and cameras - users' mental models of privacy are diverse and

\footnotetext{
${ }^{9}$ We measured participants' attention to the icon/link text in another study for the OAG [19]. Specifically, we showed participants a website screenshot and asked them a question about a nearby link, then removed the screenshot and asked them to describe any icon/link text they had noticed that would help them opt out of the sale of personal information. Less than half of the participants could accurately recall seeing the icon/link text for do-not-sell opt-outs.
}

nuanced [85]. Instead, we opted to highlight the notion of choice through the icon and use the word "privacy" in the accompanying text. As our findings show, this effectively clarified the type of choice the icon represents.

Icons should be accompanied by link texts. In line with prior work suggesting that icons and text information should appear in conjunction $[32,95,105]$, our findings show that link text has a significant impact on the icon's comprehension. Participants who saw an icon without a link text exhibited more misconceptions. Even when participants correctly recognized the concept of choice, payment, or stopping, they often failed to connect those concepts to personal information without a text description. In our icon-text combinations evaluation, conditions without link text performed comparatively worse. These findings suggest the importance of placing a descriptive link text next to an icon to aid comprehension and reduce misconceptions. This does not undermine the merits of icons - they still complement and reinforce a text description with a visual depiction, which aids recognition [51], enables textual descriptions to be more concise [38], and conveys concepts across language barriers [102]. Any icon should come with a text description when first introduced, and once it has been broadly adopted, further testing is needed to evaluate whether the text description can be removed.

Usability issues of the AdChoices icon persist despite wide adoption. Even though thousands of companies have adopted the DAA's AdChoices icon [26], our participants struggled to recognize it or accurately interpret it. In the icon pre-study, only $14 \%$ of participants recalled seeing the icon before, and even fewer correctly associated it with advertising choices. This finding echoes prior work conducted nearly a decade ago [71, 118], and shows that comprehension of this icon has not improved much since then. Coloring the AdChoices icon in green - as done by DAA's Privacy Rights icon - did not improve comprehension either. Most participants thought of "more information" upon seeing the lowercase " $i$ " or perceived the triangle shape as an audio/video play button. Icons have the potential to acquire a universal communicative power after being used over time even when their constitutive elements may not be intuitive, as demonstrated by the gear icon for settings [110] or the three arrow triangle for recycling [60]. However, our findings suggest that this is not the case for the two DAA icons, as our participants rarely associated them with privacy, do-not-sell, or other types of choices. Rather than adopting a problematic icon and expecting users will understand it over time, our findings demonstrate the importance of evaluating initial icon designs with user testing to ensure the icon is comprehensible.

Privacy choice indicators are only one component of usable privacy choices. Prior work has shown that users struggle to find privacy choices on websites $[1,47,48]$. Our research seeks to help users with this discovery problem. Our proposed icon-text combinations could serve as gateways leading users to website privacy choices, especially if a standard mechanism were to be adopted and used consistently. Nevertheless, privacy choice indicators alone are insufficient. Designing indicators to help users locate privacy choices is only the first step in improving end-to-end interactions with those choices. The indicators have to compete with many other UI elements for users' attention, and they still place the burden of accessing, learning, and exercising privacy choices on 
users $[18,66,73]$. Therefore, the interfaces users encounter after clicking on an icon/link text should be designed to minimize user effort. For instance, a web form for the CCPA do-not-sell opt-out could provide a conspicuous global "opt out" option on top, with more granular options presented below [39]. For a more substantial reduction in user burden, privacy choice indicators should be part of automated mechanisms [7, 49, 123], such as APIs that allow users to control privacy settings across websites in their web browsers, or personalized privacy assistants that learn users' privacy preferences and semi-automatically configure settings for them $[8,16,20,73]$.

\subsection{Public Policy Implications}

Incorporate user testing into the policy-making process. Researchers have argued that privacy interfaces should be developed through a user-centric and iterative design process involving user testing at early stages $[6,105,106]$. Unfortunately, most existing privacy laws either do not emphasize usability or include vague requirements for presenting privacy choices in UI design. For instance, the Federal Trade Commission (FTC) advocates that any privacy notice or choice must be "clear and prominently displayed" [120] but does not provide specific guidance on how to achieve this [114, 115]. In contrast, the widely adopted model privacy notice for US financial institutions was the product of an iterative design and testing process [43]. Another positive example is the guidance for GDPR compliance from the UK Information Commissioner's Office [56], which included visual examples to illustrate what constitutes valid consent [57]. The OAG's consideration of our research in the CCPA rule-making process further demonstrates that incorporating usertested privacy interfaces into privacy laws is not only necessary but also feasible. The OAG removed their proposed opt-out icon from the CCPA regulations [90] after we shared our findings with them about how their icon could generate critical misconceptions. Subsequently, the fourth set of modifications to the CCPA regulations recommended businesses use our blue stylized toggle icon to convey the presence of do-not-sell opt-outs [92].

Mandate unified privacy choices indicators. Even though the CCPA has an optional icon for conveying do-not-sell optouts [92], we consider it unrealistic and inefficient for privacy laws to require a specific icon or UI element for each privacy choice that businesses might offer, voluntarily or to comply with regulations. A web page with many different indicators is likely to confuse or overwhelm consumers [68]. Instead, mandating a standardized privacy choices indicator that direct users to all privacy choices in one place (e.g., a centralized privacy dashboard, account settings, or dedicated privacy choices page) would provide numerous benefits. For lawmakers, this approach is more economical compared to the significant time and resources required to develop, test, and oversee the enforcement of individual privacy choice indicators. Consumers would also appreciate a consistent and thus learnable path to navigate and exercise privacy choices [83]. Our research shows that Stylized-Toggle paired with the link text "Privacy Options" could be a good candidate for such a unified privacy choices indicator.

User-tested icons should be paired with public outreach and education. User testing can identify poor privacy choice indicators with comprehension issues, such as the DAA icons or the
OAG proposed icon [91], that would require significantly more effort in consumer education. However, even for icons that have gone through rigorous testing, consumer education is still needed to raise awareness, communicate the icon's purpose, and dispel misconceptions. In our research, even the best-performing Stylized-Toggle icon generated misconceptions occasionally. We find little documentation on associated education or public outreach efforts for most existing privacy icons. While there have been education campaigns for the AdChoices icon in the US and Europe [25, 116], consumer awareness remains low, as we and others have found [21, 118]. Whether this is due to ineffective messaging or insufficient reach is unclear. We suggest that effective education campaigns for new privacy choice icons need to address the misconceptions uncovered in initial user testing, create an active and engaging learning experience [69], and possibly use personalized education content tailoring toward individual users' characteristics [109, 121].

\section{CONCLUSION}

We conducted a series of studies to design and evaluate icons and link texts for conveying the presence of general privacy choices and the CCPA-mandated opt-out for the sale of personal information. While most icons we tested were poorly interpreted without a link text, a stylized toggle icon effectively conveyed the notion of choice and performed the best in conveying privacy choices when paired with "Privacy Options." The two CCPA-mandated link texts ("Do Not Sell My Personal Information" and "Do Not Sell My Info") accurately communicated do-not-sell opt-outs combined with most icons. Our results provide implications for designers and policymakers by highlighting the importance of accompanying icons with text descriptions, using standardized visual indicators to help users locate privacy choice mechanisms, and incorporating user testing into policy-making processes.

\section{ACKNOWLEDGMENTS}

We thank our study participants and graphic designers for their insights in generating and refining the icons, as well as the anonymous reviewers for their thoughtful feedback. This research was supported in part by a NortonLifeLock Graduate Fellowship, the Carnegie Corporation of New York, Innovators Network Foundation, grants from DARPA and AFRL under the Brandeis program (FA8750-15-2-0277), and grants from the National Science Foundation (NSF) Secure and Trustworthy Computing program (CNS-1330596, CNS-1801316, CNS-1914486). The US Government is authorized to reproduce and distribute reprints for governmental purposes not withstanding any copyright notice thereon. The views and conclusions contained herein are those of the authors and should not be interpreted as representing the official policies or endorsements, either expressed or implied, of DARPA, AFRL, NSF, or the US Government. We wish to dedicate this paper to our co-author, Prof. Joel Reidenberg, who sadly passed away in 2020.

\section{REFERENCES}

[1] Terence S Andre, H Rex Hartson, Steven M Belz, and Faith A McCreary. 2001. The User Action Framework: A Reliable Foundation for Usability Engineering Support Tools. International Fournal of Human-Computer Studies 54, 1 (2001), 107-136. https://doi.org/10.1006/ijhc.2000.0441 
[2] Annie I Antón, Julia Brande Earp, Qingfeng He, William Stufflebeam, Davide Bolchini, and Carlos Jensen. 2004. Financial Privacy Policies and the Need for Standardization. IEEE Security \& privacy 2, 2 (2004), 36-45.

[3] Apple Inc. 2021. App privacy details on the App Store. https://developer.apple. com/app-store/app-privacy-details/.

[4] Richard A Armstrong. 2014. When to Use the Bonferroni Correction. Ophthalmic and Physiological Optics 34, 5 (2014), 502-508. https://doi.org/10.1111/opo.1213

[5] Babich, Nick. 2016. UX Design: Checkbox and Toggle in Forms. https://uxplanet org/checkbox-and-toggle-in-forms-f0de6086ac41.

[6] Rebecca Balebako, Richard Shay, and Lorrie Faith Cranor. 2014. Is Your Inseam a Biometric? A Case Study on the Role of Usability Studies in Developing Public Policy. In Workshop on Usable Security (USEC). Internet Society. https: //doi.org/10.14722/usec.2014.23039

[7] Vinayshekhar Bannihatti Kumar, Roger Iyengar, Namita Nisal, Yuanyuan Feng, Hana Habib, Peter Story, Sushain Cherivirala, Margaret Hagan, Lorrie Faith Cranor, Shomir Wilson, Florian Schaub, and Norman Sadeh. 2020. Finding a Choice in a Haystack: Automatic Extraction of Opt-Out Statements from Privacy Policy Text. In The Web Conference. ACM, 1943-1954. https://doi.org/10.1145 3366423.3380262

[8] Nata M Barbosa, Joon S Park, Yaxing Yao, and Yang Wang. 2019. "What If?" Predicting Individual Users' Smart Home Privacy Preferences and Their Changes. Proceedings on Privacy Enhancing Technologies (PoPETs) 2019, 4 (2019), 211-231. https://doi.org/10.2478/popets-2019-0066

[9] Paola Benassi. 1999. TRUSTe: An Online Privacy Seal Program. Commun. ACM 42, 2 (1999), 56-59. https://doi.org/10.1145/293411.293461

[10] Adam J Berinsky, Gregory A Huber, and Gabriel S Lenz. 2012. Evaluating Online Labor Markets for Experimental Research: Amazon.com's Mechanical Turk. Political Analysis 20, 3 (2012), 351-368. https://doi.org/10.1093/pan/mpr057

[11] Jaspreet Bhatia, Travis D Breaux, Joel R Reidenberg, and Thomas B Norton. 2016. A Theory of Vagueness and Privacy Risk Perception. In Proceedings of the International Requirements Engineering Conference (RE). IEEE, 26-35. https://doi.org/10.1109/RE.2016.20

[12] Daniel Bühler, Fabian Hemmert, and Jörn Hurtienne. 2020. Universal and Intuitive? Scientific Guidelines for Icon Design. In Conference on Mensch und Computer (MuC). ACM, 91-103. https://doi.org/10.1145/3404983.3405518

[13] Michael Buhrmester, Tracy Kwang, and Samuel D Gosling. 2016. Amazon's Mechanical Turk: A New Source of Inexpensive, Yet High-Quality Data? Methodological Issues and Strategies in Clinical Research (2016), 133-139. https: //doi.org/10.1037/14805-009

[14] Simon Byers, Lorrie Faith Cranor, Dave Kormann, and Patrick McDaniel. 2004 Searching for Privacy: Design and Implementation of a P3P-Enabled Search Engine. Privacy Enhancing Technologies (PET) (2004), 314-328. https://doi.org/ $10.1007 / 11423409 \quad 20$

[15] Jacob Cohen. 2013. Statistical Power Analysis for the Behavioral Sciences. Academic press.

[16] Jessica Colnago, Yuanyuan Feng, Tharangini Palanivel, Sarah Pearman, Megan Ung, Alessandro Acquisti, Lorrie Faith Cranor, and Norman Sadeh. 2020. Informing the Design of a Personalized Privacy Assistant for the Internet of Things. In Conference on Human Factors in Computing Systems (CHI). ACM, Article 262, 13 pages. https://doi.org/10.1145/3313831.3376389

[17] Claudia Coulton and Julian Chow. 1993. Interaction Effects in Multiple Regression. Journal of Social Service Research 16, 1-2 (1993), 179-199. https: //doi.org/10.1300/J079v16n01_09

[18] Lorrie Faith Cranor. 2012. Necessary But Not Sufficient: Standardized Mechanisms for Privacy Notice and Choice. Journal on on Telecomm. \& High Tech Law 10 (2012), 273

[19] Lorrie Faith Cranor, Hana Habib, Yaxing Yao, Yixin Zou, Alessandro Acquisti, Joel Reidenberg, Norman Sadeh, and Florian Schaub. 2020. CCPA Opt-Out Icon Testing - Phase 2. Technical Report. Office of the California Attorney General. https://www.oag.ca.gov/sites/all/files/agweb/pdfs/privacy/dns-iconstudy-report-052822020.pdf.

[20] Anupam Das, Martin Degeling, Daniel Smullen, and Norman Sadeh. 2018. Personalized Privacy Assistants for the Internet of Things: Providing Users with Notice and Choice. IEEE Pervasive Computing 17, 3 (2018), 35-46. https //doi.org/10.1109/MPRV.2018.03367733

[21] Wendy Davis. 2016. Ad Industry Launches Campaign Promoting AdChoices Icon. https://www.mediapost.com/publications/article/270904/ad-industrylaunches-campaign-promoting-adchoices.html.

[22] Martin Degeling, Christine Utz, Christopher Lentzsch, Henry Hosseini, Florian Schaub, and Thorsten Holz. 2018. We Value Your Privacy...Now Take Some Cookies: Measuring the GDPR's Impact on Web Privacy. In Network and Distributed Systems Security Symposium (NDSS). Internet Society. https: //doi.org/10.14722/ndss.2019.23378

[23] Jayati Dev, Emilee Rader, and Sameer Patil. 2020. Why Johnny Can't Unsub scribe: Barriers to Stopping Unwanted Email. In Conference on Human Factors in Computing Systems (CHI). ACM, Article 38, 12 pages. https://doi.org/10.1145/ 3313831.3376165
[24] Digital Advertising Alliance. 2009. Self-Regulatory Principles for Online Behavioral Advertising. http://digitaladvertisingalliance.org/principles.

[25] Digital Advertising Alliance. 2012. Campaign Informs Consumers About Interest-Based Advertising And Encourages Them to Take Control of Their Online Privacy. https://digitaladvertisingalliance.org/press-release/digitaladvertising-alliance-daa-announces- $\% \mathrm{E} 2 \% 80 \% 98$ your-adchoices $\% \mathrm{E} 2 \% 80 \% 99$ consumer-education.

[26] Digital Advertising Alliance. 2020. DAA Participating Companies \& Organizations. https://youradchoices.com/participating.

[27] Disconnect, Inc. 2014. Disconnect Privacy Icons. https://github.com/ disconnectme/privacy-icons.

[28] Serge Egelman, Raghudeep Kannavara, and Richard Chow. 2015. Is This Thing On? Crowdsourcing Privacy Indicators for Ubiquitous Sensing Platforms. In Conference on Human Factors in Computing Systems (CHI). ACM, 1669-1678. https://doi.org/10.1145/2702123.2702251

[29] Serge Egelman, Janice Tsai, Lorrie Faith Cranor, and Alessandro Acquisti. 2009. Timing is Everything? The Effects of Timing and Placement of Online Privacy Indicators. In Conference on Human Factors in Computing Systems (CHI). ACM, 319-328. https://doi.org/10.1145/1518701.1518752

[30] Pardis Emami-Naeini, Yuvraj Agarwal, Lorrie Faith Cranor, and Hanan Hibshi. 2020. Ask the Experts: What Should Be on an IoT Privacy and Security Label?. In Symposium on Security and Privacy (S\&P). IEEE, 771-788. https://doi.org/10. 1109/sp40000.2020.00043

[31] Pardis Emami-Naeini, Henry Dixon, Yuvraj Agarwal, and Lorrie Faith Cranor. 2019. Exploring How Privacy and Security Factor into IoT Device Purchase Behavior. In Conference on Human Factors in Computing Systems (CHI). ACM, Article 534, 12 pages. https://doi.org/10.1145/3290605.3300764

[32] Samson Esayas, Tobias Mahler, and Kevin McGillivray. 2016. Is a Picture Worth a Thousand Terms? Visualising Contract Terms and Data Protection Requirements for Cloud Computing Users. In International Conference on Web Engineering (ICWE). Springer, 39-56. https://doi.org/10.1007/978-3-319-46963-8_4

[33] European Parliament. 2016. Regulation (EU) 2016/679 of the European Parliament and of the Council. https://eur-lex.europa.eu/legal-content/EN/TXT/ PDF/?uri=CELEX:32016R0679.

[34] Benjamin Fabian, Tatiana Ermakova, and Tino Lentz. 2017. Large-Scale Readability Analysis of Privacy Policies. In International Conference on Web Intelligence (WI). ACM, 18-25. https://doi.org/10.1145/3106426.3106427

[35] Federal Trade Commission. 2009. CAN-SPAM Act: A Compliance Guide for Business. https://www.ftc.gov/tips-advice/business-center/guidance/can-spamact-compliance-guide-business.

[36] Federal Trade Commission. 2017. Children's Online Privacy Protection Rule: A Six-Step Compliance Plan for Your Business. https://www.ftc.gov/tipsadvice/business-center/guidance/childrens-online-privacy-protection-rulesix-step-compliance.

[37] Adrienne Porter Felt, Robert W Reeder, Alex Ainslie, Helen Harris, Max Walker, Christopher Thompson, Mustafa Embre Acer, Elisabeth Morant, and Sunny Consolvo. 2016. Rethinking Connection Security Indicators. In Symposium on Usable Privacy and Security (SOUPS). USENIX, 1-14. https://www.usenix.org/ system/files/conference/soups2016/soups2016-paper-porter-felt.pdf.

[38] Simone Fischer-Hübner, Erik Wästlund, and Harald Zwingelberg. 2010. UI Prototypes: Policy Administration and Presentation-Version 1. (2010). http://primelife.ercim.eu/images/stories/deliverables/d4.3.1-ui_prototypespolicy_administration_and_presentation_v1.pdf.

[39] Kim Flaherty. 2018. Top 10 Design Mistakes in the Unsubscribe Experience. https://www.nngroup.com/articles/unsubscribe-mistakes/.

[40] Joseph L Fleiss, Bruce Levin, and Myunghee Cho Paik. 2013. Statistical Methods for Rates and Proportions. John Wiley \& Sons.

[41] Batya Friedman, David Hurley, Daniel C Howe, Edward Felten, and Helen Nissenbaum. 2002. Users' Conceptions of Web Security: A Comparative Study. In Conference on Human Factors in Computing Systems (CHI): Extended Abstracts. ACM, 746-747. https://doi.org/10.1145/506443.506577

[42] Stacia Garlach and Daniel Suthers. 2018. I'm Supposed to See That? AdChoices Usability in the Mobile Environment. In Hawaii International Conference on System Sciences (HICSS). Article 3779, 10 pages. https://doi.org/10.24251/hicss. 2018.476

[43] Loretta Garrison, Manoj Hastak, Jeanne M Hogarth, Susan Kleimann, and Alan S Levy. 2012. Designing Evidence-based Disclosures: A Case Study of Financial Privacy Notices. Fournal of Consumer Affairs 46, 2 (2012), 204-234. https: //doi.org/10.1111/j.1745-6606.2012.01226.x

[44] Ghostery. 2017. Ghostery: Online Privacy Made Easy. https://www.ghostery. com.

[45] Julia Gideon, Lorrie Faith Cranor, Serge Egelman, and Alessandro Acquisti. 2006. Power Strips, Prophylactics, and Privacy, Oh My!. In Symposium on Usable privacy and security (SOUPS). ACM, 133-144. https://doi.org/10.1145/1143120. 1143137

[46] Global Privacy Enforcement Network. 2017. User Controls Over Personal information. http://www.astrid-online.it/static/upload/2017/2017-gpen-sweep--international-report1.pdf. 
[47] Hana Habib, Sarah Pearman, Jiamin Wang, Yixin Zou, Alessandro Acquisti, Lorrie Faith Cranor, Norman Sadeh, and Florian Schaub. 2020. "It's a Scavenger Hunt”: Usability of Websites' Opt-Out and Data Deletion Choices. In Conference on Human Factors in Computing Systems (CHI). ACM, Article 384, 12 pages. https://doi.org/10.1145/3313831.3376511

[48] Hana Habib, Yixin Zou, Aditi Jannu, Neha Sridhar, Chelse Swoopes, Alessandro Acquisti, Lorrie Faith Cranor, Norman Sadeh, and Florian Schaub. 2019. An Empirical Analysis of Data Deletion and Opt-out Choices on 150 Websites. In Symposium on Usable Privacy and Security (SOUPS). USENIX, 387-406. https: //www.usenix.org/system/files/soups2019-habib.pdf.

[49] Hamza Harkous, Kassem Fawaz, Rémi Lebret, Florian Schaub, Kang G. Shin, and Karl Aberer. 2018. Polisis: Automated Analysis and Presentation of Privacy Policies Using Deep Learning. In USENIX Security Symposium. 531-548. https:// www.usenix.org/system/files/conference/usenixsecurity18/sec18-harkous.pdf

[50] Leif-Erik Holtz, Katharina Nocun, and Marit Hansen. 2010. Towards Displaying Privacy Information with Icons. In Privacy and Identity Management for Life. Springer, 338-348. https://doi.org/10.1007/978-3-642-20769-3_27

[51] William K Horton. 1994. The Icon Book: Visual Symbols for Computer Systems and Documentation. John Wiley \& Sons, Inc.

[52] Connor Huff and Dustin Tingley. 2015. "Who Are These People?” Evaluating the Demographic Characteristics and Political Preferences of MTurk Survey Respon dents. Research \& Politics 2, 3 (2015). https://doi.org/10.1177/2053168015604648

[53] IAB Europe. 2011. IAB EU Framework for Online Behavioural Advertis ing. https://www.edaa.eu/wp-content/uploads/2012/10/2013-11-11-IABEurope-OBA-Framework_.pdf.

[54] IAB Europe. 2019. GDPR Transparency and Consent Framework. https: //iabtechlab.com/standards/gdpr-transparency-and-consent-framework/.

[55] Renato Iannella and Adam Finden. 2010. Privacy Awareness: Icons and Expression for Social Networks. In International Workshop for Technical, Economic and Legal Aspects of Business Models for Virtual Goods. Article 1, 15 pages. http://virtualgoods.org/2010/VirtualGoodsBook2010_13.pdf.

[56] Information Commissioner's Office. 2019. Guide to the General Data Protection Regulation (GDPR). https://ico.org.uk/for-organisations/guide-to-dataprotection/guide-to-the-general-data-protection-regulation-gdpr/.

[57] Information Commissioner's Office. 2019. What is valid consent? https://ico.org.uk/for-organisations/guide-to-data-protection/guide-tothe-general-data-protection-regulation-gdpr/consent/what-is-valid-consent/.

[58] Sarah J Isherwood, Siné JP McDougall, and Martin B Curry. 2007. Icon Identification in Context: The Changing Role of Icon Characteristics with User Experience Human Factors 49, 3 (2007), 465-476. https://doi.org/10.1518/001872007X200102

[59] Carlos Jensen and Colin Potts. 2004. Privacy Policies as Decision-Making Tools: An Evaluation of Online Privacy Notices. In Conference on Human Factors in Computing Systems (CHI). ACM, 471-478. https://doi.org/10.1145/985692.985752

[60] Penny Jones and Jerry Powell. 1999. Gary Anderson Has Been Found! Resource Recycling 18 (1999), 25-27. https://discardstudies.com/wp-content/uploads/ 2012/07/garyandersonfound.pdf.

[61] Michiel de Jong, Jan-Christoph Borchardt, Hugo Roy, Ian McGowan, Jimm Stout, Suzanne Azmayesh, Christopher Talib, Vincent Tunru, Madeline O'Leary, and Evan Mullen. 2020. Terms of Service; Didn't Read. https://tosdr.org/en/ frontpage.

[62] Saraschandra Karanam, Janhavi Viswanathan, Anand Theertha, Bipin Indurkhya, and Herre Van Oostendorp. 2010. Impact of Placing Icons Next to Hyperlinks on Information-Retrieval Tasks on the Web. In Proceedings of the Annual Meeting of the Cognitive Science Society, Vol. 32. eScholarship, 2834-2839. https://escholarship.org/content/qt27w0n9kc/qt27w0n9kc.pdf.

[63] Patrick Gage Kelley, Joanna Bresee, Lorrie Faith Cranor, and Robert W Reeder 2009. A "Nutrition Label" for Privacy. In Symposium on Usable Privacy and Security (SOUPS). Article 4, 12 pages. https://doi.org/10.1145/1572532.1572538

[64] Patrick Gage Kelley, Lucian Cesca, Joanna Bresee, and Lorrie Faith Cranor 2010. Standardizing Privacy Notices: An Online Study of the Nutrition Label Approach. In Conference on Human factors in Computing Systems (CHI). ACM, 1573-1582. https://doi.org/10.1145/1753326.1753561

[65] Patrick Gage Kelley, Lorrie Faith Cranor, and Norman Sadeh. 2013. Privacy as Part of the App Decision-Making Process. In Conference on Human Factors in Computing Systems (CHI). ACM, 3393-3402. https://doi.org/10.1145/2470654. 2466466

[66] Jennifer King. 2020. CCPA Comments, Round Two: The Battle of the Do-Not-Sell Button. https://cyberlaw.stanford.edu/blog/2020/02/ccpa-comments-roundtwo-battle-do-not-sell-button.

[67] Saranga Komanduri, Richard Shay, Greg Norcie, and Blase Ur. 2011. AdChoices? Compliance with Online Behavioral Advertising Notice and Choice Require ments. ISfLP 7 (2011), 603.

[68] Stefan Korff and Rainer Böhme. 2014. Too Much Choice: End-user Privacy Decisions in the Context of Choice Proliferation. In Symposium On Usable Privacy and Security (SOUPS). USENIX, 69-87. https://www.usenix.org/system/ files/soups14-paper-korff.pdf.

[69] Lauren I Labrecque, Ereni Markos, and Aron Darmody. 2019. Addressing Online Behavioral Advertising and Privacy Implications: A Comparison of Passive
Versus Active Learning Approaches. Fournal of Marketing Education (2019), 1-16. https://doi.org/10.1177/0273475319828788

[70] Jonathan Lazar, Jinjuan Heidi Feng, and Harry Hochheiser. 2017. Research Methods in Human-Computer Interaction. Morgan Kaufmann.

[71] Pedro Giovanni Leon, Justin Cranshaw, Lorrie Faith Cranor, Jim Graves, Manoj Hastak, Blase Ur, and Guzi Xu. 2012. What Do Online Behavioral Advertising Privacy Disclosures Communicate to Users?. In Workshop on Privacy in the Electronic Society (WPES). ACM, 19-30. https://doi.org/10.1145/2381966.2381970

[72] Eric Lin, Saul Greenberg, Eileah Trotter, David Ma, and John Aycock. 2011. Does Domain Highlighting Help People Identify Phishing Sites?. In Conference on Human Factors in Computing Systems (CHI). ACM, 2075-2084. https://doi.org/ 10.1145/1978942.1979244

[73] Bin Liu, Mads Schaarup Andersen, Florian Schaub, Hazim Almuhimedi, Shikun Aerin Zhang, Norman Sadeh, Yuvraj Agarwal, and Alessandro Acquisti. 2016. Follow My Recommendations: A Personalized Privacy Assistant for Mobile App Permissions. In Symposium on Usable Privacy and Security (SOUPS). USENIX, 27-41. https://www.usenix.org/system/files/conference/soups2016/soups2016paper-liu.pdf.

[74] Dominique Machuletz and Rainer Böhme. 2020. Multiple Purposes, Multiple Problems: A User Study of Consent Dialogs after GDPR. Proceedings on Privacy Enhancing Technologies (PoPETs) 2020, 2 (2020), 481-498. https://doi.org/10. 2478/popets-2020-0037

[75] Maureen Mahoney. 2020. California Consumer Privacy Act: Are Consumers' Digital Rights Protected? Technical Report. Consumer Reports. https://advocacy.consumerreports.org/wp-content/uploads/2020/09/ CR CCPA-Are-Consumers-Digital-Rights-Protected 092020 vf.pdf.

[76] Manfredo Massironi. 2001. The Psychology of Graphic Images: Seeing, Drawing, Communicating. Psychology Press.

[77] Aleecia M McDonald and Lorrie Faith Cranor. 2008. The Cost of Reading Privacy Policies. I/S: A Journal of Law and Policy for the Information Society 4 (2008), 543.

[78] Aleecia M McDonald and Lorrie Faith Cranor. 2010. Americans' Attitudes about Internet Behavioral Advertising Practices. In Workshop on Privacy in the Electronic Society (WPES). ACM, 63-72. https://doi.org/10.1145/1866919.1866929

[79] Aleecia M Mcdonald, Robert W Reeder, Patrick Gage Kelley, and Lorrie Faith Cranor. 2009. A Comparative Study of Online Privacy Policies and Formats. Proceedings on Privacy Enhancing Technologies (PoPETs) (2009), 37-55. https: //doi.org/10.1007/978-3-642-03168-7_3

[80] George R Milne and Mary J Culnan. 2004. Strategies for Reducing Online Privacy Risks: Why Consumers Read (Or Don't Read) Online Privacy Notices. Journal of Interactive Marketing 18, 3 (2004), 15-29.

[81] Mozilla. 2020. Privacy Icons. https://wiki.mozilla.org/Privacy_Icons.

[82] Network Advertising Initiative. 2018. NAI Code of Conduct. https://www. networkadvertising.org/sites/default/files/nai_code2018.pdf.

[83] Don Norman. 2013. The Design of Everyday Things. Basic books.

[84] Midas Nouwens, Ilaria Liccardi, Michael Veale, David Karger, and Lalana Kagal. 2020. Dark Patterns after the GDPR: Scraping Consent Pop-Ups and Demonstrating Their Influence. In Conference on Human Factors in Computing Systems (CHI). ACM, Article 194, 13 pages. https://doi.org/10.1145/3313831.3376321

[85] Maggie Oates, Yama Ahmadullah, Abigail Marsh, Chelse Swoopes, Shikun Zhang, Rebecca Balebako, and Lorrie Faith Cranor. 2018. Turtles, Locks, and Bathrooms: Understanding Mental Models of Privacy through Illustration. Proceedings on Privacy Enhancing Technologies (PoPETs) 2018, 4 (2018), 5-32. https://doi.org/10.1515/popets-2018-0029

[86] Jonathan A Obar and Anne Oeldorf-Hirsch. 2020. The Biggest Lie on the Internet: Ignoring the Privacy Policies and Terms of Service Policies of Social Networking Services. Information, Communication \& Society 23, 1 (2020), 128147. https://doi.org/10.1080/1369118X.2018.1486870

[87] Sean O'Connor, Ryan Nurwono, and Eleanor Birrell. $2020 . \quad$ (Un)clear and (In)conspicuous: The right to opt-out of sale under CCPA. arXiv preprint:2009.07884 (2020).

[88] Office of the California Attorney General. 2019. California Consumer Privacy Act (CCPA): Proposed Text of Regulations. https://www.oag.ca.gov/sites/all/ files/agweb/pdfs/privacy/ccpa-proposed-regs.pdf.

[89] Office of the California Attorney General. 2019. The California Privacy Rights and Enforcement Act of 2020. https://oag.ca.gov/system/files/initiatives/pdfs/19$0017 \% 20 \% 28$ Consumer\%20Privacy\%20\%29.pdf.

[90] Office of the California Attorney General. 2020. California Consumer Privacy Act (CCPA): Final Text of Proposed Regulations. https://oag.ca.gov/sites/all/ files/agweb/pdfs/privacy/oal-sub-final-text-of-regs.pdf.

[91] Office of the California Attorney General. 2020. California Consumer Privacy Act (CCPA): First Set of Modified Regulations. https://oag.ca.gov/sites/all/files/ agweb/pdfs/privacy/ccpa-text-of-mod-clean-020720.pdf

[92] Office of the California Attorney General. 2020. California Consumer Privacy Act (CCPA): Fourth Set of Modified Regulations. https://oag.ca.gov/sites/all/ files/agweb/pdfs/privacy/ccpa-prop-mods-text-of-regs-4th.pdf.

[93] Office of the California Attorney General. 2021. California Consumer Privacy Act (CCPA) Current Rulemaking Activities. https://oag.ca.gov/privacy/ccpa/regs. 
[94] Online Trust Alliance. 2018. Email Marketing \& Unsubscribe Audit. https://www.internetsociety.org/wp-content/uploads/2019/04/2018-emailunsubscribe-report.pdf.

[95] Stefania Passera. 2015. Beyond the Wall of Text: How Information Design Can Make Contracts User-Friendly. In International Conference of Design, User Experience, and Usability (DUXU). Springer, 341-352. https://doi.org/10.1007/ 978-3-319-20898-5 33

[96] Peter Peduzzi, John Concato, Elizabeth Kemper, Theodore R Holford, and Alvan R Feinstein. 1996. A Simulation Study of the Number of Events per Variable in Logistic Regression Analysis. Journal of Clinical Epidemiology 49, 12 (1996), 1373-1379. https://doi.org/10.1016/S0895-4356(96)00236-3

[97] Rebecca S Portnoff, Linda N Lee, Serge Egelman, Pratyush Mishra, Derek Leung, and David Wagner. 2015. Somebody's Watching me? Assessing the Effectiveness of Webcam Indicator Lights. In Conference on Human Factors in Computing Systems (CHI). ACM, 1649-1658. https://doi.org/10.1145/2702123.2702164

[98] PrivacyGrade.org. 2020. PrivacyGrade.org. http://privacygrade.org/home.

[99] Elissa M Redmiles, Sean Kross, and Michelle L Mazurek. 2019. How Well Do My Results Generalize? Comparing Security and Privacy Survey Results from MTurk, Web, and Telephone Samples. In Symposium on Security and Privacy (S\&P). IEEE, 1326-1343. https://doi.org/10.1109/SP.2019.00014

[100] Joel R Reidenberg, Travis Breaux, Lorrie Faith Cranor, Brian French, Amanda Grannis, James T Graves, Fei Liu, Aleecia McDonald, Thomas B Norton, and Rohan Ramanath. 2015. Disagreeable Privacy Policies: Mismatches Between Meaning and Users' Understanding. Berkeley Technology Law fournal 30 (2015), 39. https://doi.org/10.15779/Z384K33

[101] Joel R Reidenberg, N Cameron Russell, Vlad Herta, William Sierra-Rocafort, and Thomas B Norton. 2018. Trustworthy Privacy Indicators: Grades, Labels Certifications, and Dashboards. Washington University Law Review 96, Article 1409 (2018).

[102] Arianna Rossi and Monica Palmirani. 2019. DaPIS: a Data Protection Icon Set to Improve Information Transparency under the GDPR. Knowledge of the Law in the Big Data Age 252 (2019), 181-195.

[103] Jeffrey N Rouder, Christopher R Engelhardt, Simon McCabe, and Richard D Morey. 2016. Model Comparison in ANOVA. Psychonomic Bulletin \& Review 23 , 6 (2016), 1779-1786. https://doi.org/10.3758/s13423-016-1026-5

[104] Johnny Saldaña. 2015. The Coding Manual for Qualitative Researchers. Sage.

[105] Florian Schaub, Rebecca Balebako, Adam L. Durity, and Lorrie Faith Cranor. 2015. A Design Space for Effective Privacy Notices. In Symposium On Usable Privacy and Security (SOUPS). USENIX, Article 1,17 pages. https://www.usenix. org/system/files/conference/soups2015/soups15-paper-schaub.pdf.

[106] Florian Schaub and Lorrie Faith Cranor. 2020. Usable and Useful Privacy Inter faces. In An Introduction to Privacy for Technology Professionals, Travis Breaux (Ed.). IAPP, 176-299.

[107] Florian Schaub, Aditya Marella, Pranshu Kalvani, Blase Ur, Chao Pan, Emily Forney, and Lorrie Faith Cranor. 2017. Watching Them Watching Me: Browser Extensions' Impact on User Privacy Awareness and Concern. In Workshop on Usable Security and Privacy (USEC). Internet Society. https://doi.org/10.14722/ usec.2016.23017

[108] Stuart E Schechter, Rachna Dhamija, Andy Ozment, and Ian Fischer. 2007. The Emperor's New Security Indicators. In Symposium on Security and Privacy (S\&P). IEEE, 51-65. https://doi.org/10.1109/SP.2007.35

[109] Kristina L Schmid, Susan E Rivers, Amy E Latimer, and Peter Salovey. 2008. Targeting or Tailoring? Maximizing Resources to Create Effective Health Communications. Marketing Health Services 28, 1 (2008), 32.

[110] Johanna M Silvennoinen, Tuomo Kujala, and Jussi PP Jokinen. 2017. Semantic Distance as A Critical Factor in Icon Design for In-Car Infotainment Systems. Applied Ergonomics 65 (2017), 369-381. https://doi.org/10.1016/j.apergo.2017.07. 014

[111] Stanford Legal Design Lab. 2020. Icons for legal help. https://betterinternet. law.stanford.edu/design-guide/icons-for-legal-help/.

[112] The Digital Advertising Alliance. 2020. License the Privacy Rights Icon. https: //digitaladvertisingalliance.org/license-pricon.

[113] The Digital Advertising Alliance. 2020. Your AdChoices. https://youradchoices $\operatorname{com} /$.

[114] The Federal Trade Commission. 2012. Protecting Consumer Privacy in an Era of Rapid Change: A Proposed Framework for Businesses and Policymakers. https://www.ftc.gov/sites/default/files/documents/reports/federaltrade-commission-report-protecting-consumer-privacy-era-rapid-changerecommendations/120326privacyreport.pdf.

[115] The Federal Trade Commission. 2013. .com Disclosures: How to Make Effective Disclosures in Digital Advertising. https://www.ftc.gov/sites/default/files/ attachments/press-releases/ftc-staff-revises-online-advertising-disclosureguidelines/130312dotcomdisclosures.pdf

[116] TrustArc. 2013. EDAA rolls out pan-European consumer education campaign on OBA. https://trustarc.com/blog/2013/06/13/edaa-rolls-out-pan-europeanconsumer-education-campaign-on-oba/.

[117] Janice Y Tsai, Serge Egelman, Lorrie Faith Cranor, and Alessandro Acquisti. 2011. The Effect of Online Privacy Information on Purchasing Behavior: An
Experimental Study. Information Systems Research 22, 2 (2011), 254-268. https: //doi.org/10.1287/isre.1090.0260

[118] Blase Ur, Pedro Giovanni Leon, Lorrie Faith Cranor, Richard Shay, and Yang Wang. 2012. Smart, Useful, Scary, Creepy: Perceptions of Online Behavioral Advertising. In Symposium on Usable Privacy and Security (SOUPS). ACM, Article 4, 15 pages. https://doi.org/10.1145/2335356.2335362

[119] Christine Utz, Martin Degeling, Sascha Fahl, Florian Schaub, and Thorsten Holz. 2019. (Un)informed Consent: Studying GDPR Consent Notices in the Field. In Conference on Computer and Communications Security (CCS). ACM, 973-990. https://doi.org/10.1145/3319535.3354212

[120] Ari Ezra Waldman. 2018. Privacy, Notice, and Design. Stanford Technology Law Review 21 (2018), 74

[121] Logan Warberg, Alessandro Acquisti, and Douglas Sicker. 2019. Can Privacy Nudges be Tailored to Individuals' Decision Making and Personality Traits?. In Workshop on Privacy in the Electronic Society (WPES). ACM, 175-197. https: //doi.org/10.1145/3338498.3358656

[122] Susan Wiedenbeck. 1999. The Use of Icons and Labels in an End User Application Program: an Empirical Study of Learning and Retention. Behaviour \& Information Technology 18, 2 (1999), 68-82. https://doi.org/10.1080/014492999119129

[123] Shomir Wilson, Florian Schaub, Frederick Liu, Kanthashree Mysore Sathyendra, Daniel Smullen, Sebastian Zimmeck, Rohan Ramanath, Peter Story, Fei Liu, Norman Sadeh, and Noah A Smith. 2018. Analyzing Privacy Policies at Scale: From Crowdsourcing to Automated Annotations. ACM Transactions on the Web (TWEB) 13, Article 1 (2018). https://doi.org/10.1145/3230665

\section{A SURVEY QUESTIONS}

\section{A.1 Icon Design Evaluation}

Please answer the following questions with regards to the displayed symbol [and phrase]. Make sure not to reveal any private or personally identifiable information about yourself or others in your responses to any open-ended questions.

[The symbol or symbol/phrase condition to which the participant was randomly assigned is displayed to the participant.]

(1) What, if anything, does this [symbol/symbol and phrase] communicate to you? Please be as complete as possible. (Open-ended response)

(2) Imagine if you saw this [symbol/symbol and phrase] on a website. What do you think would happen if you clicked on this [symbol/symbol and phrase]? (Open-ended response)

[The DAA's blue AdChoices icon is displayed]

(3) Have you ever seen this symbol on a website before?

- Yes

- No

- I am not sure

(4) Imagine if you saw this symbol on a website. What do you think would happen if you clicked on this symbol?

[Present the icon set in randomized order.]

(5) Which of these symbols do you think best conveys that there's an option to tell websites "do not sell my personal information?" [The order of Q5/6 and Q7/8 was randomized for the icon refinement testing.]

(6) Please explain why you selected the icon above. (Open-ended response) [Present the icon set in randomized order.]

(7) Which of these symbols do you think best conveys that there's an option to make choices about the use of your personal information?

(8) Please explain why you selected the icon above. (Open-ended response)

(9) Are you aware of any laws in the United States that require companies to provide a "do not sell my personal information" option? 
- No

- Yes (please name or describe them):

(10) What is your age?

- 18-24

- 25-34

- $35-44$

- $45-54$

- 55-64

- $65-74$

- 75-84

- 85 or older

- Prefer not to answer

(11) What is your gender?

- Women

- Men

- Non-binary

- Prefer to self describe:

- Prefer not to answer

(12) What is the highest level of education you have completed?

- Less than high school

- High school degree or equivalent

- Some college, no degree

- Associate's degree, occupational

- Associate's degree, academic

- Bachelor's degree

- Master's degree

- Professional degree

- Doctoral degree

- Prefer not to answer

(13) What was your total household income before taxes during the past 12 months?

- Under $\$ 15,000$

- $\$ 15,000$ to $\$ 24,999$

- $\$ 25,000$ to $\$ 34,999$

- $\$ 35,000$ to $\$ 49,999$

- $\$ 50,000$ to $\$ 74,999$

- $\$ 75,000$ to $\$ 99,999$

- $\$ 100,000$ to $\$ 149,999$

- $\$ 150,000$ or above

- Prefer not to answer

(14) In which state do you currently reside? (Drop-down list)

(15) Which of the following best describes your educational background or job field?

- I have an education in, or work in, the field of computer science, computer engineering or IT.

- I do not have an education in, or work in, the field of computer science, computer engineering or IT.

- Prefer not to answer

(16) If you have any feedback on the survey, please leave it here. (Open-ended response)

\section{A.2 Link Text Evaluation}

Please answer the following questions with regards to the web link. Make sure not to reveal any private or personally identifiable information about yourself or others in your responses to any openended questions.

Imagine if you saw this web link on a website.
[The link text condition to which the participant was randomly assigned is displayed to the participant.]

(1) What types of ["selling" / "personal information" / "choices" / "options" / "opt-outs"] do you think this link refers to? (Open-ended response, displayed only if the participant saw a link text that includes the respective element)

(2) What do you think would happen if you clicked on this link?

(3) Which of the following do you think could happen if you clicked this symbol/link on a web page [For each statement below, participants were asked to choose from a 5-point likert scale "Definitely" "Probably" "Not sure" "Probably not" and "Definitely not." Statements were presented in randomized order.]

- It will take me to the website's Terms of Service statement.

- It will take me to a page that verifies that the website does not sell my personal information.

- It will take me to a page where I can pay to protect my personal information.

- It will take me to a page with choices about the sale of my personal information.

- It will immediately communicate to the website that I do not want my personal information to be sold.

- It will take me to a page with choices about how my personal information is used and shared by the website.

- It will give the website permission to sell my personal information.

- It will take me to a warning not to share my personal information with websites.

(4) Are you aware of any laws in the United States that require companies to provide a "do not sell my personal information" option?

- No

- Yes (please name or describe them):

(5) What is your age? (See Appendix A.1 for answer options)

(6) What is your gender? (See Appendix A.1 for answer options)

(7) What is the highest level of education you have completed? (See Appendix A.1 for answer options)

(8) What was your total household income before taxes during the past 12 months? (See Appendix A.1 for answer options)

(9) In which state do you currently reside? (Drop-down list)

(10) Which of the following best describes your educational background or job field? (See Appendix A.1 for answer options)

(11) Which of the following best describes your primary occupation?

- Administrative Support (e.g., secretary assistant)

- Art, Writing, or Journalism (e.g., author, reporter, sculptor)

- Business, Management, or Financial (e.g., manager, accountant, banker)

- Education or Science ( e.g., teacher, professor, scientist)

- Legal (e.g., lawyer, paralegal)

- Medical ( e.g., doctor, nurse, dentist)

- Computer Engineering or IT Professional (e.g., programmer or IT consultant)

- Engineer in other field (e.g., civil or bio engineer)

- Service (e.g., retail clerk, server)

- Skilled Labor (e.g., electrician, plumber, carpenter) 
- Unemployed

- Retired

- College student

- Graduate student

- Mechanical Turk worker

- Other:

- Prefer not to answer

(12) If you have any feedback on the survey, please leave it here. (Open-ended response)

\section{A.3 Icon-Text Combinations Evaluation}

Please answer the following questions with regards to the [symbol/link/symbol and link] in the rectangular highlighted area near the bottom of the web page displayed. Make sure not to reveal any private or personally identifiable information about yourself or others in your responses to any open-ended questions.

[Display the screenshot of the web page with the icon, link text, or icon-text combination that the participant was randomly assigned to. Below is an example of one study condition]

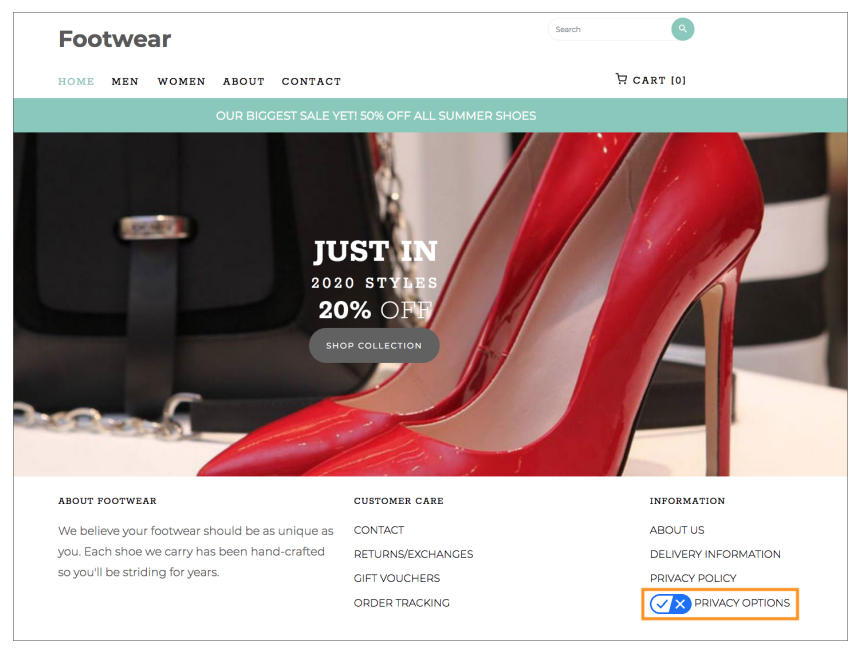

Close up of highlighted area:

\section{PRIVACY OPTIONS}

(1) What do you think would happen if you clicked on the [symbol/link/symbol and link] in the highlighted area on this web page?

[Display close up of highlighted area again.]

(2) What do you think ["sell" / "info" / "information" / "choices" / "options" / "opt-outs"] refers to in this link? (Open-ended response, displayed only if the participant saw a link text that includes the respective element) [Display close up of highlighted area again.]

(3) Which of the following do you think could happen if you clicked this symbol/link on a web page? [For each statement below, participants were asked to choose from a 5-point likert scale "Definitely" "Probably" "Not sure" "Probably not" and "Definitely not." Statements were presented in randomized order.]

- It will take me to a page where I can update the information in my user profile on the website.

- It will take me to a page with choices about how my personal information is used and shared by the website.

- It will take me to a page with choices about the sale of my personal information.

- It will take me to a page with more information about how the company uses and shares the personal information it collects about me.

- It will cause the website to send unwanted emails.

- It will give the website permission to sell my personal information.

- It will take me to a page with ads about privacy and security products.

- It will take me to a page that steals my information or has a virus or malware.

(4) Are you aware of any laws in the United States that require companies to provide a "do not sell my personal information" option?

- No

- Yes (please name or describe them):

(5) What is your age? (See Appendix A.1 for answer options)

(6) What is your gender? (See Appendix A.1 for answer options)

(7) What is the highest level of education you have completed? (See Appendix A.1 for answer options)

(8) What was your total household income before taxes during the past 12 months? (See Appendix A.1 for answer options)

(9) In which state do you currently reside? (Drop-down list)

(10) Which of the following best describes your educational background or job field? (See Appendix A.1 for answer options)

(11) Which of the following best describes your primary occupation? (See Appendix A.2 for answer options)

(12) If you have any feedback on the survey, please leave it here. (Open-ended response)

\section{A.4 CCPA Toggle Icon Evaluation}

Please answer the following questions with regards to the symbol and link in the rectangular highlighted area near the bottom of the web page displayed. Make sure not to reveal any private or personally identifiable information about yourself or others in your responses to any open-ended questions.

[Display the screenshot of the web page with the icon and "Do Not Sell My Personal Information" link text that the participant was randomly assigned to.]

[Display close up of highlighted area.]

(1) What do you think would happen if you clicked on the symbol and link in the highlighted area on this web page? [Display close up of highlighted area again.]

(2) What do you think ["sell"/"information"] refers to in this link? (Open-ended response) [Display close up of highlighted area again.]

(3) Which of the following do you think could happen if you clicked this symbol/link on a web page? [For each statement 
below, participants were asked to choose from a 5-point likert scale "Definitely" "Probably" "Not sure" "Probably not" and "Definitely not." Statements were presented in randomized order.]

- It will immediately change the setting on this website from "Do Not Sell My Personal Information" to "Sell My Personal Information."

- It will immediately change the setting on this website from "Sell My Personal Information" to "Do Not Sell My Personal Information."

- It will take me to a page where I can choose whether or not the website can sell my personal information.

- It will take me to a page where I can confirm that I do not want my personal information to be sold by the website.

- It will take me to a page with more information about how the website uses and shares my personal information.

- It will cause the website to send me unwanted emails.

- It will take me to a page with ads about privacy and security products.
- It will take me to a page that steals my information or has a virus or malware.

(4) Are you aware of any laws in the United States that require companies to provide a "do not sell my personal information" option?

- No

- Yes (please name or describe them):

(5) What is your age? (See Appendix A.1 for answer options)

(6) What is your gender? (See Appendix A.1 for answer options)

(7) What is the highest level of education you have completed? (See Appendix A.1 for answer options)

(8) What was your total household income before taxes during the past 12 months? (See Appendix A.1 for answer options)

(9) In which state do you currently reside? (Drop-down list)

(10) Which of the following best describes your educational background or job field? (See Appendix A.1 for answer options)

(11) Which of the following best describes your primary occupation? (See Appendix A.2 for answer options)

(12) If you have any feedback on the survey, please leave it here. (Open-ended response) 


\section{B PARTICIPANT DEMOGRAPHICS}

\begin{tabular}{|c|c|c|c|c|c|c|}
\hline $\begin{array}{l}\text { Sample Size } \\
\text { Invalid Responses }\end{array}$ & $\begin{array}{c}\text { Icon } \\
\text { Preliminary } \\
240 \\
11\end{array}$ & $\begin{array}{c}\text { Icon } \\
\text { Refinement } \\
280 \\
0\end{array}$ & $\begin{array}{c}\text { Link Text } \\
\text { Preliminary } \\
140 \\
9\end{array}$ & $\begin{array}{c}\text { Link Text } \\
\text { Refinement } \\
400 \\
0\end{array}$ & Combination & $\begin{array}{c}\text { Toggle } \\
421 \\
18\end{array}$ \\
\hline \multicolumn{7}{|l|}{ Age } \\
\hline $18-24$ & $5.00 \%$ & $5.71 \%$ & $8.57 \%$ & $7.00 \%$ & $10.29 \%$ & $12.11 \%$ \\
\hline $25-34$ & $45.00 \%$ & $45.71 \%$ & $52.14 \%$ & $49.00 \%$ & $35.76 \%$ & $45.13 \%$ \\
\hline $35-44$ & $29.58 \%$ & $29.64 \%$ & $22.86 \%$ & $23.25 \%$ & $25.95 \%$ & $23.04 \%$ \\
\hline $45-54$ & $12.08 \%$ & $10.00 \%$ & $8.57 \%$ & $11.00 \%$ & $15.74 \%$ & $11.16 \%$ \\
\hline $55-64$ & $7.08 \%$ & $6.79 \%$ & $4.29 \%$ & $7.00 \%$ & $8.72 \%$ & $6.18 \%$ \\
\hline$>65$ & $1.25 \%$ & $2.14 \%$ & $3.57 \%$ & $2.75 \%$ & $3.13 \%$ & $1.90 \%$ \\
\hline Prefer Not to Answer & $0.00 \%$ & $0.00 \%$ & $0.00 \%$ & $0.00 \%$ & $0.41 \%$ & $0.28 \%$ \\
\hline \multicolumn{7}{|l|}{ Gender } \\
\hline Female & $41.25 \%$ & $44.64 \%$ & $34.29 \%$ & $39.50 \%$ & $46.87 \%$ & $47.51 \%$ \\
\hline Male & $58.33 \%$ & $55.36 \%$ & $64.29 \%$ & $60.00 \%$ & $51.98 \%$ & $50.83 \%$ \\
\hline Non-binary & $0.00 \%$ & $0.00 \%$ & $1.43 \%$ & $0.50 \%$ & $0.41 \%$ & $0.95 \%$ \\
\hline Self-described & $0.00 \%$ & $0.00 \%$ & $0.00 \%$ & $0.00 \%$ & $0.14 \%$ & $0.00 \%$ \\
\hline Prefer Not to Answer & $0.42 \%$ & $0.00 \%$ & $0.00 \%$ & $0.00 \%$ & $0.61 \%$ & $0.71 \%$ \\
\hline \multicolumn{7}{|l|}{ Education } \\
\hline Less than High School & $0.83 \%$ & $0.71 \%$ & $0.71 \%$ & $0.25 \%$ & $0.54 \%$ & $0.48 \%$ \\
\hline High School & $9.58 \%$ & $13.57 \%$ & $7.86 \%$ & $13.50 \%$ & $8.92 \%$ & $12.11 \%$ \\
\hline Some College & $15.83 \%$ & $18.57 \%$ & $17.14 \%$ & $18.00 \%$ & $21.93 \%$ & $18.76 \%$ \\
\hline Associate's, Occupational & $5.83 \%$ & $8.21 \%$ & $4.29 \%$ & $7.75 \%$ & $6.81 \%$ & $5.23 \%$ \\
\hline Associate's, Academic & $1.67 \%$ & $5.00 \%$ & $4.29 \%$ & $4.75 \%$ & $6.40 \%$ & $5.46 \%$ \\
\hline Bachelor & $49.58 \%$ & $42.86 \%$ & $51.43 \%$ & $43.75 \%$ & $39.03 \%$ & $43.71 \%$ \\
\hline Master & $13.33 \%$ & $8.21 \%$ & $13.57 \%$ & $11.00 \%$ & $11.92 \%$ & $9.26 \%$ \\
\hline Professional & $2.50 \%$ & $1.79 \%$ & $0.71 \%$ & $0.50 \%$ & $1.63 \%$ & $2.38 \%$ \\
\hline Doctoral & $0.42 \%$ & $1.07 \%$ & $0.00 \%$ & $0.50 \%$ & $2.18 \%$ & $2.14 \%$ \\
\hline Prefer Not to Answer & $0.42 \%$ & $0.00 \%$ & $0.00 \%$ & $0.00 \%$ & $0.61 \%$ & $0.48 \%$ \\
\hline \multicolumn{7}{|l|}{ State Residence } \\
\hline California & $11.25 \%$ & $14.29 \%$ & $20.00 \%$ & $9.75 \%$ & $10.42 \%$ & $16.39 \%$ \\
\hline Non-California & $88.75 \%$ & $85.71 \%$ & $80.00 \%$ & $90.25 \%$ & $89.58 \%$ & $83.61 \%$ \\
\hline \multicolumn{7}{|c|}{ Educational/Job Background related to CS/IT } \\
\hline Yes & $38.75 \%$ & $27.50 \%$ & $47.86 \%$ & $33.00 \%$ & $23.02 \%$ & $30.64 \%$ \\
\hline No & $56.67 \%$ & $66.79 \%$ & $47.86 \%$ & $62.75 \%$ & $72.55 \%$ & $63.66 \%$ \\
\hline Prefer Not to Answer & $4.58 \%$ & $5.71 \%$ & $4.29 \%$ & $4.25 \%$ & $4.43 \%$ & $5.70 \%$ \\
\hline \multicolumn{7}{|c|}{ Awareness of any U.S. laws that require companies to provide a "do not sell my personal information" option } \\
\hline Yes & $4.58 \%$ & $4.64 \%$ & $2.86 \%$ & $3.00 \%$ & $9.81 \%$ & $7.13 \%$ \\
\hline No & $95.42 \%$ & $95.36 \%$ & $97.14 \%$ & $97.00 \%$ & $90.19 \%$ & $92.87 \%$ \\
\hline
\end{tabular}

Table 5: Age, gender, education, state residence demographics of participants as well as their familiarity with CCPA in each study. 


\section{REGRESSION OUTPUTS}

\section{C.1 Icon-Text Combinations Evaluation}

\begin{tabular}{|c|c|c|c|c|c|c|c|c|c|c|c|c|}
\hline & \multicolumn{3}{|c|}{ Choice } & \multicolumn{3}{|c|}{ Privacy } & \multicolumn{3}{|c|}{ Misconception } & \multicolumn{3}{|c|}{ Do-Not-Sell } \\
\hline & $\beta$ & S.E. & Adj. $p$ & $\beta$ & S.E. & Adj. $p$ & $\beta$ & S.E. & Adj. $p$ & $\beta$ & S.E. & Adj. $p$ \\
\hline Intercept & .52 & .31 & 1.0 & 2.0 & .41 & $<.001^{*}$ & -3.1 & .74 & $.001^{*}$ & -.06 & .33 & 1.0 \\
\hline \multicolumn{13}{|c|}{ Condition (ref = Toggle-Privacy Options, None-Do Not Sell My Personal Information for Do-Not-Sell) } \\
\hline Toggle-None & -1.0 & .40 & .16 & -1.2 & .48 & .20 & 2.1 & .80 & .21 & & & \\
\hline Toggle-Do Not Sell My P.I. & -.62 & .40 & 1.0 & -4.0 & .58 & $<.001^{*}$ & 2.4 & .79 & .06 & .26 & .40 & 1.0 \\
\hline Toggle-Personal Info Choices & -1.2 & .40 & .06 & -2.4 & .47 & $<.001^{*}$ & 2.8 & .78 & $.009^{*}$ & -3.2 & .66 & $<.001^{*}$ \\
\hline Toggle-Do Not Sell My Info & -.33 & .40 & 1.0 & -4.3 & .60 & $<.001^{*}$ & 1.5 & .82 & .77 & .52 & .39 & 1.0 \\
\hline Toggle-Privacy Choices & -.23 & .40 & 1.0 & -.86 & .48 & .85 & -1.6 & .82 & .76 & 2.2 & .49 & $<.001^{*}$ \\
\hline Toggle-Privacy Options & & & & & & & & & & -4.3 & 1.0 & $.001^{*}$ \\
\hline DAA-None & -3.6 & .60 & $<.001^{*}$ & -3.0 & .49 & $<.001^{*}$ & 2.2 & .79 & .14 & & & \\
\hline DAA-Do Not Sell My P.I. & -.49 & .40 & 1.0 & -2.6 & .47 & $<.001^{*}$ & .81 & .89 & 1.0 & -.09 & .38 & 1.0 \\
\hline DAA-Privacy Options & .29 & .42 & 1.0 & -.26 & .52 & 1.0 & -.02 & 1.0 & 1.0 & -4.3 & 1.0 & $.001^{*}$ \\
\hline DAA-Personal Info Choices & -1.6 & .41 & $.004^{*}$ & -2.4 & .48 & $<.001^{*}$ & 2.5 & .79 & $.04^{*}$ & -2.6 & .59 & $<.001^{*}$ \\
\hline DAA-Do Not Sell My Info & -.59 & .40 & 1.0 & -3.5 & .52 & $<.001^{*}$ & 1.3 & .84 & 1.0 & .25 & .39 & 1.0 \\
\hline DAA-Privacy Choices & .10 & .41 & 1.0 & -.67 & .49 & 1.0 & .06 & 1.0 & 1.0 & -2.4 & .51 & $<.001^{*}$ \\
\hline Dollar-None & -3.0 & .50 & $<.001^{*}$ & -5.3 & .82 & $<.001^{*}$ & 4.2 & .78 & $<.001^{*}$ & & & \\
\hline Dollar-Do Not Sell My P.I. & -.92 & .39 & .32 & -3.6 & .52 & $<.001^{*}$ & 1.6 & .82 & .78 & .02 & .38 & 1.0 \\
\hline Dollar-Privacy Options & -.28 & .40 & 1.0 & -.52 & .51 & 1.0 & 1.7 & .81 & .53 & -2.1 & .49 & $<.001^{*}$ \\
\hline Dollar-Personal Info Choices & -1.3 & .40 & $.03^{*}$ & -2.0 & .46 & $<.001^{*}$ & 2.9 & .78 & $.005^{*}$ & -2.8 & .59 & $<.001^{*}$ \\
\hline Dollar-Do Not Sell My Info & -.47 & .40 & 1.0 & -3.7 & .53 & $<.001^{*}$ & -.55 & 1.2 & 1.0 & .91 & .41 & .36 \\
\hline Dollar-Privacy Choices & -1.1 & .39 & .10 & -.82 & .48 & .91 & 2.0 & .79 & .21 & -2.7 & .59 & $<.001^{*}$ \\
\hline None-Do Not Sell My P.I. & -.94 & .40 & .32 & -3.3 & .51 & $<.001^{*}$ & 1.2 & .84 & 1.0 & & & \\
\hline None-Privacy Options & -.48 & .40 & 1.0 & -.65 & .49 & 1.0 & .03 & 1.0 & 1.0 & -2.8 & .59 & $<.001^{*}$ \\
\hline None-Personal Info Choices & -1.3 & .40 & $.02^{*}$ & -2.0 & .46 & $<.001^{*}$ & 2.8 & .78 & $.008^{*}$ & -2.8 & .59 & $<.001^{*}$ \\
\hline None-Do Not Sell My Info & .10 & .43 & 1.0 & -3.4 & .52 & $<.001^{*}$ & -.58 & 1.2 & 1.0 & .62 & .40 & 1.0 \\
\hline None-Privacy Choices & -.64 & .40 & 1.0 & -.94 & .49 & .71 & .50 & .94 & 1.0 & -2.4 & .55 & $<.001^{*}$ \\
\hline Icon-None & & & & & & & & & & -4.6 & .76 & $<.001$ \\
\hline \multicolumn{13}{|l|}{ Age $($ ref = 18-34) } \\
\hline $35-54$ & .42 & .13 & $.02^{*}$ & -.07 & .15 & 1.0 & -.18 & .18 & 1.0 & .29 & .18 & 1.0 \\
\hline$\geq 55$ & .35 & .20 & 1.0 & -.42 & .22 & .74 & .43 & .25 & .97 & .58 & .27 & .36 \\
\hline \multicolumn{13}{|l|}{ Gender $($ ref = Female $)$} \\
\hline Male & .10 & .12 & 1.0 & .18 & .14 & -.16 & .17 & 1.0 & 1.0 & -.11 & .17 & 1.0 \\
\hline \multicolumn{13}{|c|}{ Technical expertise $($ ref $=$ None $)$} \\
\hline Yes & -.42 & .15 & .13 & -.11 & .17 & 1.0 & .46 & .20 & .32 & -.68 & .22 & $.02^{*}$ \\
\hline \multicolumn{13}{|c|}{ Highest obtained education $($ ref = No college degree) } \\
\hline College degree & .33 & .14 & .27 & -.13 & .15 & 1.0 & -.46 & .18 & .22 & .20 & .19 & 1.0 \\
\hline Graduate degree & .31 & .19 & 1.0 & .11 & .21 & 1.0 & -.61 & .26 & .32 & .39 & .26 & 1.0 \\
\hline
\end{tabular}

Table 6: Regression results for the four binary dependent variables (conveys choice, privacy, misconceptions, or do-not-sell choices) coded from participants' open-ended expectations. Due to perfect separation in the DAA-none and Dollar-none conditions, the icon-only conditions were collapsed together (Icon-None) for the do-not-sell choices regression. For each regression term we provide the estimate of the coefficient $(\beta)$, the standard error, and p-value adjusted with the Holm-Bonferroni correction. A significant negative coefficient indicates that participants in that group were less likely to have the expectation represented by the dependent variable, relative to the reference baseline. $\left(^{*}\right)$ marks significant results for $\alpha=.05$. 


\begin{tabular}{|c|c|c|c|c|c|c|c|c|c|}
\hline & \multicolumn{3}{|c|}{ Do-Not-Sell Choices } & \multicolumn{3}{|c|}{ Give Sell Permission } & \multicolumn{3}{|c|}{ Privacy Choices } \\
\hline & $\beta$ & S.E. & Adj. $p$ & $\beta$ & S.E. & Adj. $p$ & $\beta$ & S.E. & Adj. $p$ \\
\hline Intercept & .41 & .31 & 1.0 & -2.0 & .44 & $<.001^{*}$ & 2.5 & .54 & $<.001^{*}$ \\
\hline \multicolumn{10}{|c|}{ Condition (ref = None-Do Not Sell My Personal Information, Toggle-Privacy Options for Privacy Choices) } \\
\hline Toggle-None & -.98 & .39 & .33 & .56 & .53 & 1.0 & -1.8 & .60 & .07 \\
\hline Toggle-Do Not Sell My P.I. & -.22 & .40 & 1.0 & 1.7 & .49 & $.02^{*}$ & -2.1 & .59 & $.009^{*}$ \\
\hline Toggle-Personal Info Choices & -.87 & .39 & .60 & .96 & .50 & 1.0 & -1.7 & .60 & .10 \\
\hline Toggle-Do Not Sell My Info & -.55 & .39 & 1.0 & .65 & .52 & 1.0 & -1.8 & .60 & .07 \\
\hline Toggle-Privacy Choices & -.09 & .39 & 1.0 & 1.4 & .49 & .12 & -1.1 & .62 & .99 \\
\hline Toggle-Privacy Options & .07 & .39 & 1.0 & .87 & .51 & 1.0 & & & \\
\hline DAA-None & -2.9 & .52 & $<.001^{*}$ & -.45 & .62 & 1.0 & -2.4 & .58 & $.001^{*}$ \\
\hline DAA-Do Not Sell My P.I. & -.19 & .39 & 1.0 & .11 & .56 & 1.0 & -.73 & .65 & 1.0 \\
\hline DAA-Privacy Options & -.56 & .38 & 1.0 & .93 & .50 & 1.0 & -.62 & .66 & 1.0 \\
\hline DAA-Personal Info Choices & -.20 & .40 & 1.0 & 1.2 & .50 & .47 & -1.8 & .60 & .06 \\
\hline DAA-Do Not Sell My Info & .18 & .41 & 1.0 & -.22 & .59 & 1.0 & -1.6 & .61 & .19 \\
\hline DAA-Privacy Choices & -.23 & .39 & 1.0 & .85 & .51 & 1.0 & -.25 & .70 & 1.0 \\
\hline Dollar-None & -1.3 & .39 & $.04^{*}$ & -.38 & .62 & 1.0 & -3.6 & .60 & $<.001^{*}$ \\
\hline Dollar-Do Not Sell My P.I. & -.29 & .39 & 1.0 & .12 & .56 & 1.0 & -1.7 & .59 & .06 \\
\hline Dollar-Privacy Options & -.05 & .40 & 1.0 & .48 & .53 & 1.0 & -.69 & .66 & 1.0 \\
\hline Dollar-Personal Info Choices & -.36 & .39 & 1.0 & .56 & .53 & 1.0 & -1.1 & .63 & 1.0 \\
\hline Dollar-Do Not Sell My Info & -.14 & .39 & 1.0 & -.58 & .66 & 1.0 & -1.3 & .61 & .52 \\
\hline Dollar-Privacy Choices & -.65 & .38 & 1.0 & .57 & .51 & 1.0 & -1.4 & .60 & .36 \\
\hline None-Privacy Options & -.20 & .39 & 1.0 & .82 & .51 & 1.0 & -.42 & .68 & 1.0 \\
\hline None-Personal Info Choices & -.84 & .39 & .72 & -.09 & .57 & 1.0 & -1.7 & .60 & .08 \\
\hline None-Do Not Sell My Info & .84 & .45 & 1.0 & .11 & .57 & 1.0 & -.70 & .66 & 1.0 \\
\hline None-Privacy Choices & -.24 & .40 & 1.0 & .55 & .53 & 1.0 & -.52 & .68 & 1.0 \\
\hline None-Do Not Sell My P.I. & & & & & & & -.86 & .65 & 1.0 \\
\hline \multicolumn{10}{|l|}{ Age $($ ref $=18-34)$} \\
\hline $35-54$ & .09 & .12 & 1.0 & -.04 & .15 & 1.0 & .27 & .15 & 1.0 \\
\hline$\geq 55$ & .17 & .19 & 1.0 & -.008 & .23 & 1.0 & .16 & .23 & 1.0 \\
\hline \multicolumn{10}{|l|}{ Gender (ref = Female) } \\
\hline Male & -.02 & .12 & 1.0 & -.13 & .15 & 1.0 & -.21 & .14 & 1.0 \\
\hline \multicolumn{10}{|c|}{ Technical expertise $($ ref $=$ None $)$} \\
\hline Yes & .03 & .15 & 1.0 & .72 & .17 & $<.001^{*}$ & -.03 & .18 & 1.0 \\
\hline \multicolumn{10}{|c|}{ Highest obtained education $($ ref $=$ No college degree) } \\
\hline College degree & .31 & .13 & .51 & -.09 & .16 & 1.0 & .15 & .16 & 1.0 \\
\hline Graduate degree & .17 & .18 & 1.0 & .07 & .22 & 1.0 & .63 & .24 & .15 \\
\hline
\end{tabular}

Table 7: Regression results for the scenarios: It will take me to a page with choices about the sale of my personal information" (Do-Not-Sell Choices), "It will give the website permission to sell my personal information" (Give Sell Permission), and "It will take me to a page with choices about how my personal information is used and shared by the website" (Privacy Choices). For each regression term we provide the estimate of the coefficient $(\beta)$, the standard error, and p-value adjusted with the HolmBonferroni correction. A significant negative coefficient indicates that participants in that group were less likely to have the expectation represented by the dependent variable, relative to the reference baseline. $\left({ }^{*}\right)$ marks significant results for $\alpha=.05$. 


\section{C.2 OAG Toggle Evaluation}

\begin{tabular}{|c|c|c|c|c|c|c|}
\hline & \multicolumn{3}{|c|}{ Misconception } & \multicolumn{3}{|c|}{ Toggle Control } \\
\hline & $\beta$ & S.E. & $p$ & $\beta$ & S.E. & $p$ \\
\hline Intercept & -1.2 & .34 & $<.001^{*}$ & -1.6 & .36 & $<.001^{*}$ \\
\hline \multicolumn{7}{|c|}{ Condition $($ style ref $=$ Stylized Toggle $;$ color ref $=$ Blue $)$} \\
\hline CalAG & .83 & .28 & $.003^{*}$ & .87 & .30 & $.003^{*}$ \\
\hline CalAG-X & .86 & .28 & $.003^{*}$ & .87 & .30 & $.004^{*}$ \\
\hline Red & .003 & .22 & .99 & .28 & .23 & .23 \\
\hline \multicolumn{7}{|l|}{ Age $($ ref = 18-34) } \\
\hline $35-54$ & -.24 & .24 & .31 & -.15 & .25 & .54 \\
\hline$\geq 55$ & -.99 & .49 & $.04^{*}$ & -1.2 & .58 & $.03^{*}$ \\
\hline \multicolumn{7}{|c|}{ Gender $($ ref $=$ Female $)$} \\
\hline Male & -.08 & .23 & .73 & .03 & .24 & .89 \\
\hline \multicolumn{7}{|c|}{ Technical expertise $($ ref $=$ None $)$} \\
\hline Yes & -.47 & .25 & .06 & -.86 & .27 & $.002^{*}$ \\
\hline \multicolumn{7}{|c|}{ Highest obtained education (ref = No college degree) } \\
\hline College degree & .65 & .26 & $.01^{*}$ & .68 & .27 & $.01^{*}$ \\
\hline Graduate degree & .50 & .36 & .17 & .71 & .38 & .06 \\
\hline
\end{tabular}

Table 8: Regression results for the binary dependent variables: conveys a misconception and perceived as a toggle control, coded from participants' open-ended expectations. We report results from the main effect model with icon style and color as the main independent variables, as the interaction between icon style and color was not significant. For each regression term we provide the estimate of the coefficient $(\beta)$, the standard error, and $\mathbf{p}$-value adjusted with the Holm-Bonferroni correction. A significant positive coefficient indicates that participants in that group were more likely to have the expectation represented by the dependent variable, relative to the reference baseline. $\left(^{*}\right)$ marks significant results for $\alpha=.05$.

\begin{tabular}{|c|c|c|c|c|c|c|}
\hline & \multicolumn{3}{|c|}{ Do-Not-Sell Switch } & \multicolumn{3}{|c|}{ Do-Not-Sell Choices } \\
\hline & $\beta$ & S.E. & Adj. $p$ & $\beta$ & S.E. & Adj. $p$ \\
\hline Intercept & -.77 & .35 & $.03^{*}$ & .70 & .31 & $.03^{*}$ \\
\hline \multicolumn{7}{|c|}{ Condition $($ style ref $=$ Stylized Toggle; color ref $=$ Blue) } \\
\hline CalAG & .73 & .38 & .05 & -.92 & .27 & $<.001^{*}$ \\
\hline CalAG-X & 1.0 & .39 & $.009^{*}$ & -.88 & .27 & $.001^{*}$ \\
\hline Red & 1.0 & .37 & $.006^{*}$ & .14 & .22 & .51 \\
\hline $\mathrm{CalAG}^{*} \mathrm{Red}$ & -1.2 & .52 & $.02^{*}$ & & & \\
\hline CalAG-X*Red & -1.8 & .53 & $<.001^{*}$ & & & \\
\hline \multicolumn{7}{|l|}{ Age $($ ref $=18-34)$} \\
\hline $35-54$ & .24 & .23 & .31 & .04 & .23 & .87 \\
\hline$\geq 55$ & .29 & .39 & .46 & .60 & .41 & .14 \\
\hline \multicolumn{7}{|c|}{ Gender $($ ref $=$ Female $)$} \\
\hline Male & .04 & .22 & .84 & -.53 & .22 & $.02^{*}$ \\
\hline \multicolumn{7}{|c|}{ Technical expertise $($ ref $=$ None $)$} \\
\hline Yes & .21 & .24 & .37 & -.35 & .24 & .15 \\
\hline \multicolumn{7}{|c|}{ Highest obtained education $($ ref $=$ No college degree) } \\
\hline College degree & -.15 & .24 & .52 & -.006 & .24 & .98 \\
\hline Graduate degree & -.07 & .34 & .83 & -.08 & .35 & .83 \\
\hline
\end{tabular}

Table 9: Regression results for the scenarios:"It will immediately change the setting on this website from 'Sell My Personal Information' to 'Do Not Sell My Personal Information' " (Do-Not-Sell Switch), and "It will take me to a page with choices about the sale of my personal information" (Do-NotSell Choices). We report results from the main effect model for Do-Not-Sell Choices with icon style and color as the main independent variables, as the interaction between icon style and color was not significant. For each regression term we provide the estimate of the coefficient $(\beta)$, the standard error, and p-value adjusted with the Holm-Bonferroni correction. A significant positive coefficient indicates that participants in that group were more likely to have the expectation represented by the dependent variable, relative to the reference baseline. $\left({ }^{*}\right)$ marks significant results for $\alpha=.05$. 